\title{
ASYMPTOTIC FORMULAS FOR TOEPLITZ DETERMINANTS
}

BY

ESTELLE BASOR

ABsTRACT. The object of this paper is to find an asymptotic formula for determinants of finite dimensional Toeplitz operators generated by a class of functions with singularities. The formula is a generalization of the Strong Szegö Limit Theorem.

1. Introduction. For a function $\sigma \in L_{1}\left(S^{1}\right)$ consider the family of matrices

$$
T_{n}[\sigma]=\left(c_{j k}\right), \quad j, k=0, \ldots, n,
$$

where

$$
c_{j k}=\frac{1}{2 \pi} \int_{-\pi}^{\pi} e^{-i(j-k) \theta} \sigma(\theta) d \theta .
$$

The matrices $T_{n}[\sigma]$ are called Toeplitz matrices and their determinants $D_{n}[\sigma]$ are called Toeplitz determinants.

The asymptotic behavior of these determinants has been the object of study by many people for some time. The basic problem has been to determine the asymptotic behavior given a set of conditions for $\sigma$.

The first main result was proved by Szegö $[8, \S 5.5]$. He originally proved that if $\sigma$ was positive and had a derivative satisfying a Lipschitz condition, then for $D_{n}[\sigma]$ one has the asymptotic formula

$$
D_{n}[\sigma] \sim E[\sigma] G[\sigma]^{n+1}, \quad n \rightarrow \infty .
$$

The factors $E[\sigma]$ and $G[\sigma]$ are defined by

$$
G[\sigma]=\exp s_{0}, \quad E[\sigma]=\exp \sum_{k=1}^{\infty} k s_{k} s_{-k},
$$

where

$$
\log \sigma(\theta)=\sum_{k=-\infty}^{\infty} s_{k} e^{i k \theta} .
$$

(1.1) has since been shown to be valid for $\sigma$ 's satisfying weaker conditions We mention here only that it was proved by Hirschman [10] that if $\sigma$ satisfies

Received by the editors October 30, 1976.

AMS (MOS) subject classifications (1970). Primary 41A60, 47B35.

Key words and phrases. Asymptotic formula, Toeplitz determinant, singular generating function.

- American Mathematical Society 1978 
(i) $\sigma(\theta) \neq 0$,

(ii) $\Delta_{-\pi \leqslant \theta \leqslant \pi} \arg \sigma(\theta)=0$,

(iii) $\sum_{-\infty}^{\infty}\left|c_{k}\right|+\left[\sum_{-\infty}^{\infty}|k|\left|c_{k}\right|^{2}\right]^{1 / 2}<\infty$

then (1.1) holds.

We will, however, be concerned with $\sigma$ 's where this formula breaks down. To be more precise, suppose

$$
\sigma(\theta)=\tau(\theta) \prod_{r=1}^{R}\left(2-2 \cos \left(\theta-\theta_{r}\right)\right)^{\alpha_{r}}\left(-e^{i\left(\theta-\theta_{r}\right)}\right)^{\beta_{r}}
$$

where

(i) $\left|\arg \left(-e^{i\left(\theta-\theta_{r}\right)}\right)\right|<\pi$;

(ii) $\tau(\theta)$ satisfies (1.2(i), (ii));

(iii) $\tau(\theta)$ has a derivative satisfying a Lipschitz condition with some positive exponent;

(iv) $R \alpha_{r}>-\frac{1}{2}$.

The asymptotic behavior for this $\sigma(\theta)$ was investigated by Hartwig and Fischer [6]. They conjectured that the correct asymptotic formula takes the form

$$
E\left[\tau, \alpha_{1}, \ldots, \alpha_{r}, \beta_{1}, \ldots, \beta_{r}, \theta_{1}, \ldots, \theta_{r}\right] \times n^{\Sigma\left(\alpha_{r}^{2}-\beta_{r}^{2}\right)} G[\tau]^{n+1}
$$

where $E\left[\tau, \alpha_{1}, \ldots, \alpha_{r}, \theta_{1}, \ldots, \theta_{r}\right]$ is some constant. They were able to verify this in the case $\tau(\theta)=1, R=1$ and $\alpha_{r}=0$. Moreover, they gave a heuristic argument leading to a conjecture concerning the form of the constant in the general case. More will be said about this later. Lenard [12] conjectured the same result with $\alpha_{r}>-\frac{1}{2}$ and $\beta_{r}=0$, and he was able to prove his conjecture in the case $\tau(\theta)=1, R=2, \theta_{1}=0$ and $\theta_{2}=\pi$.

A much more complete result was obtained by Widom [16] who showed that if $\beta_{r}=0$ then (1.4) holds. We should also remark here that in all of the above special cases the constant was determined.

In [16], Widom also proved (1.4) with $R=1, \alpha, \beta$ real, and $|\alpha|,|\beta|<\frac{1}{2}$, but did not determine the constant.

We shall prove (1.4) in this paper, with the additional restriction that $\beta_{r}$ is purely imaginary. Without this restriction, the arguments used in [16] for $R=1, \alpha, \beta$ real, and $|\alpha|,|\beta|<\frac{1}{2}$ do not extend to more than one point of singularity.

Before we state the exact values of the constant, let us make the following observations. It is no loss of generality to assume that $G[\sigma]=1$. We will do this for the rest of the paper. And with our assumptions (1.2(i), (ii)) for $\tau(\theta)$ we have a factorization 


$$
\tau(\theta)=\tau_{+}(\theta) \tau_{-}(\theta) \quad \text { with } \tau_{+}^{ \pm 1} \in H_{\infty}, \quad \tau_{-}^{ \pm 1} \in \bar{H}_{\infty} .
$$

Recall $H_{p}$ is the set of $L_{p}$ functions on the unit circle whose Fourier coefficients vanish for negative values of the index. $\bar{H}_{p}$ is the set of functions $\bar{f}$ where $f \in H_{p}$. We define the factors $\tau_{+}$and $\tau_{-}$by

$$
\tau_{+}(\theta)=\exp \left[\sum_{1}^{\infty} s_{k} e^{i k \theta}\right], \quad \tau_{-}(\theta)=\exp \left[\sum_{0}^{\infty} s_{-k} e^{-i k \theta}\right],
$$

where

$$
\log \tau(\theta)=\sum_{-\infty}^{\infty} s_{k} e^{i k \theta}
$$

Here we choose any continuous logarithm, and since $G[\tau]=1$, we may assume $s_{0}=0$. The constant in (1.4) is then

$$
\begin{array}{r}
E[\tau] \prod_{r \neq s}\left(1-e^{i\left(\theta_{s}-\theta_{r}\right)}\right)^{-\left(\alpha_{r}+\beta_{r}\right)\left(\alpha_{s}-\beta_{s}\right)} \\
\times \prod_{r} \tau_{+}\left(\theta_{r}\right)^{-\alpha_{r}+\beta_{r}} \tau_{-}\left(\theta_{r}\right)^{-\alpha_{r}-\beta_{r}} \prod_{r} E_{\alpha_{r}, \beta_{r}} \\
\text { where }\left(\left|\arg \left(1-e^{i\left(\theta_{s}-\theta_{r}\right)}\right)\right|<\pi / 2\right) .
\end{array}
$$

Here

$$
E_{\alpha, \beta}=G(1+\alpha+\beta) G(1+\alpha-\beta) / G(1+2 \alpha),
$$

where $G$ is the Barnes $G$-function [1].

We shall now outline the sections of this paper. In $\$ 2$ it is shown that for $\left|R \alpha_{r}\right|<\frac{1}{2}$ and $\beta$ imaginary, the limit of $D_{n}[\sigma] n^{-\sum_{r-1}^{R}\left(\alpha_{r}^{2}-\beta_{r}^{2}\right)}$ exists. The propositions in this section are taken from [16] but with certain modifications. The restriction to imaginary $\beta$ actually makes things considerably easier.

In $\$ 3$ a preliminary form of the asymptotic formula is determined. The problem of finding the constant in the asymptotic formula is reduced to finding one for

$$
\sigma(\theta)=(2-2 \cos \theta)^{\alpha}\left(-e^{i \theta}\right)^{\beta} .
$$

In this section we restrict ourselves to $\alpha_{r}$ real and $\left|\alpha_{r}\right|<\frac{1}{2}$. The reason we can do this is the following. Suppose we think of $\alpha_{r}$ as fixed and think of $D_{n}[\sigma]$ as a function of the $\alpha_{r}$ 's. It is easy to see that $D_{n}[\sigma]$ is an analytic function of the $\alpha_{r}$ 's for $R \alpha_{r}>-\frac{1}{2}$. Hence $D_{n}[\sigma] n^{-\Sigma\left(\alpha_{r}^{2}-\beta_{r}^{2}\right)}$ (written $S_{n}[\sigma]$ in what follows) is a family of analytic functions. In $\$ 5$ we will show that the family is uniformly bounded on compact sets in $R \alpha_{r}>-\frac{1}{2}$. Hence, by the Vitali convergence theorem and the existence of the limit already established for $\left|R \alpha_{r}\right|<\frac{1}{2}$, we will have the existence of the limit for $\alpha_{r}$ satisfying $R \alpha_{r}>-\frac{1}{2}$, and this limit must be an analytic function in the $\alpha_{r}$ 's. Thus we can say that when we are 
concerned with the constant in (1.4) it is enough to consider $\alpha_{r}$ real and $\left|\alpha_{r}\right|<\frac{1}{2}$.

With these restrictions we obtain a preliminary form of the asymptotic formula. In \$3, computations that lead to this preliminary form contain uniform asymptotic formulas for orthogonal polynomials with a weight function of the form $\sigma$. This is of independent interest. We should also remark that the techniques used here are all borrowed from Widom [16], and this preliminary form verifies the conjecture of Hartwig and Fischer.

In $\$ 4$ we consider the function

$$
\sigma(\theta)=(2-2 \cos \theta)^{\alpha}\left(-e^{i \theta}\right)^{\beta}, \quad|R \alpha|<\frac{1}{2} .
$$

Let $D_{n}[\alpha]$ denote $D_{n}[\sigma]$ for the above $\sigma$. In $\S 4$ it is shown that

$$
\lim _{n \rightarrow \infty} \frac{D_{n-1}[\alpha+1] n^{-2 \alpha-1}}{D_{n}[\alpha]}=\frac{\Gamma(1+\alpha+\beta) \Gamma(1+\alpha-\beta)}{\Gamma(1+2 \alpha) \Gamma(2+2 \alpha)} .
$$

This implies that the factor $E_{\alpha, \beta}$ in (1.5) satisfies the difference equation

$$
E_{\alpha+1, \beta}=\frac{\Gamma(1+\alpha+\beta) \Gamma(1+\alpha-\beta)}{\Gamma(1+2 \alpha) \Gamma(2+2 \alpha)} E_{\alpha, \beta} .
$$

We should remark here that this is not a surprising result. Leonard showed that if $\beta=0$ then

$$
E_{\alpha, \beta}=G(1+\alpha)^{2} / G(1+2 \alpha),
$$

where $G$ is the Barnes $G$-function [1] mentioned previously. It is also easy to show from the computation of Hartwig and Fischer that if $\alpha=0$ then

$$
E_{\alpha, \beta}=G(1+\beta) G(1-\beta) \text {. }
$$

Since $G(1)=1$ one might guess

$$
E_{\alpha, \beta}=G(1+\alpha+\beta) G(1+\alpha-\beta) / G(1+2 \alpha) \text {. }
$$

Now the Barnes $G$-function is an entire function of order 2 with the following properties:

(i) $G(-n)=0, n=0,1,2,3, \ldots$,

(ii) $G(-n)$ is a zero of order $n+1$,

(iii) $G(z+1)=\Gamma(z) G(z)$.

If the guess is right the difference equation should follow.

$\$ 5$ ties the loose ends together. Here it is shown that $S_{n}[\sigma]$ is uniformly bounded on compact sets in $R \alpha>-\frac{1}{2}$. This might seem an awkward place to include this result, but the proof follows naturally after the difference equation section and we would be confronted with too much repetition otherwise.

Then it is shown that $E_{\alpha, \beta}$ is actually $G(1+\alpha+\beta) G(1+\alpha-\beta) / G(1+$ 
$2 \alpha$ ). This is really not too difficult once one has the difference equation.

We end this introduction by stating some notation that will be used throughout the paper. $T_{n}[\sigma]$ can be thought of as an operator on a space of sequences $\left\{a_{0}, \ldots, a_{n}\right\}$ or the space of polynomials $a_{0}+\cdots+a_{n} z^{n}$ of degree at most $n$. The latter interpretation will be used most frequently.

We should also note here that $\sigma$ can be written as

$$
\tau(\theta) \prod_{r=1}^{R}\left(1-\frac{z}{z_{r}}\right)^{\alpha_{r}+\beta_{r}}\left(1-\frac{z_{r}}{z}\right)^{\alpha_{r}-\beta_{r}}
$$

where $z=e^{i \theta}$ and $z_{r}=e^{i \theta_{r}}$. Here $\left(1-z / z_{r}\right)^{\alpha_{r}+\beta_{r}}$ denotes the limit on the unit circle of that branch of the function which is analytic in $|z|<1$ and takes the value of 1 at $z=0$. The term $\left(1-z_{r} / z\right)^{\alpha_{r}-\beta_{r}}$ is defined similarly. Both of these representations will be used.

The last representation allows us to write $\sigma(\theta)$ as a product $\sigma_{+}(\theta) \sigma_{-}(\theta)$ where

$$
\sigma_{+}(\theta)=\tau_{+}(\theta) \Pi\left(1-\frac{z}{z_{r}}\right)^{\alpha_{r}+\beta_{r}} \quad\left(z=e^{i \theta}\right)
$$

and

$$
\sigma_{-}(\theta)=\tau_{-}(\theta) \Pi\left(1-\frac{z_{r}}{z}\right)^{\alpha_{r}-\beta_{r}} \quad\left(z=e^{i \theta}\right) .
$$

Here $\tau_{+}(\theta)$ and $\tau_{-}(\theta)$ are the factors of $\tau$ defined for (1.4). Since $G[\tau]=1$, $\sigma_{+}, \sigma_{-}, \tau_{+}$and $\tau_{-}$can all be assumed to have 1 as the constant term in their Fourier expansion.

Finally, the $k$ th Fourier coefficient of a function $f$ will be denoted by $f_{k}$.

2. Existence of $\lim S_{n}[\sigma]$ when $\left|R \alpha_{r}\right|<\frac{1}{2}$. The existence of $\lim _{n \rightarrow \infty} S_{n}[\sigma]$ can be proved by the methods of [16] with obvious modifications. In this section we shall show that for some $\delta>0$,

$$
\frac{D_{n-1}[\sigma]}{D_{n}[\sigma]}=1-\sum_{r}\left(\alpha_{r}^{2}-\beta_{r}^{2}\right)+O\left(n^{-1-\delta}\right)
$$

It is clear that (2.1) implies the existence of the above limit and we proceed to derive it.

Suppose now that the function $\sigma(\theta)$ possesses a factorization $\sigma(\theta)=$ $\sigma_{+}(\theta) \sigma_{-}(\theta)$ where $\sigma_{+}^{ \pm 1} \in H_{2}, \sigma_{-}^{ \pm 1} \in \bar{H}_{2}, \sigma_{+} / \sigma_{-} \in L_{\infty}, \sigma_{-} / \sigma_{+} \in L_{\infty}$. We define the following operators: 


$$
\begin{array}{ll}
()^{+}: L_{2} \rightarrow H_{2}, & ()^{+} \text {is projection onto } H_{2}, \\
()^{-}: L_{2} \rightarrow \bar{H}_{2}, & ()^{-} \text {is projection onto } \bar{H}_{2}, \\
()_{+}: L_{2} \rightarrow z H_{2}, & (f)_{+}=f-(f)^{-}, \\
()_{-}: L_{2} \rightarrow z \bar{H}_{2}, & (f)_{-}=f-(f)^{+}, \\
U: \overline{z H}_{2} \rightarrow z H_{2}, & U: \phi \rightarrow\left(z^{-n} \phi \sigma_{-} / \sigma_{+}\right)_{+}, \\
V: z H_{2} \rightarrow z \bar{H}_{2}, & V: \psi \rightarrow\left(z^{n} \psi \sigma_{+} / \sigma_{-}\right)_{-} .
\end{array}
$$

Notice the dependence of $U$ and $V$ on $n$. In [16] proofs are given for the following two propositions.

Proposition 2.1. If the operators $\mathrm{I}-U V: z \mathrm{H}_{2} \rightarrow z \mathrm{H}_{2}$ and $\mathrm{I}-V U$ : $\overline{z H}_{2} \rightarrow \overline{z H}_{2}$ are invertible, then $T_{n}[\sigma]$ is invertible and $p=T_{n}[\sigma]^{-1} q(p$ and $q$ are polynomials of degree at most $n$ ) is given by

$$
p=q / \sigma+\phi / \sigma_{+}+z^{n} \psi / \sigma_{-},
$$

where $\phi$ and $\psi$ are the solutions of

$$
\begin{aligned}
& (I-V U) \phi=-\left(z^{n}\left(\sigma_{+} / \sigma_{-}\right)\left(z^{-n} q / \sigma_{+}\right)^{-}\right)_{-} \\
& (I-U V) \psi=-\left(z^{-n}\left(\sigma_{-} / \sigma_{+}\right)\left(q / \sigma_{-}\right)^{+}\right)_{+} .
\end{aligned}
$$

Proposition 2.2. Assume that $\sigma_{-}$and $\sigma_{+}$have constant term equal to 1 in their Fourier expansion. Then if the operators $I-U V$ and $I-V U$ (and hence $\left.T_{n}[\sigma]\right)$ are invertible we have

$$
\frac{D_{n-1}[\sigma]}{D_{n}[\sigma]}=1+\frac{1}{2 \pi} \int_{-\pi}^{\pi} z^{n} \psi(z) \frac{\sigma_{+}(z)}{\sigma_{-}(z)} d \theta
$$

where $\psi(z)$ corresponds to $q=1$ in (2.3).

In order to apply Propositions 2.1 and 2.2 to the case in question, we use (1.6) and (1.7) for the factors $\sigma_{+}$and $\sigma_{-}$. We can also assume, as noted previously, that the factors $\sigma_{+}$and $\sigma_{-}$have constant term equal to 1 in their Fourier expansion. The rest of this section will be devoted to proving Proposition 2.3. As one can easily see, Propositions 2.2 and 2.3 yield the formula (2.1).

Proposition 2.3. For sufficiently large $n,(I-U V)^{-1}$ and $(I-V U)^{-1}$ exist (and hence $T_{n}[\sigma]^{-1}$ ), and for some $\delta>0$ we have

$$
\frac{1}{2 \pi} \int_{-\pi}^{\pi} z^{n} \psi(z) \frac{\sigma_{+}(z)}{\sigma_{-}(z)} d \theta=-\sum_{r}\left(\alpha_{r}^{2}-\beta_{r}^{2}\right) n^{-1}+O\left(n^{-1-\delta}\right),
$$

where $\psi(z)$ corresponds to $q=1$ in (2.3).

We begin by considering the operator $K_{\alpha}: L_{2}(0, \infty) \rightarrow L_{2}(0, \infty)$ where $K_{\alpha}$ has kernel 


$$
\begin{aligned}
K_{\alpha}(x, y)= & \pi^{-2} \Gamma(1+2 \beta) \Gamma(1-2 \beta) \sin \pi(\alpha+\beta) \sin \pi(\alpha-\beta) \\
& \times \int_{0}^{\infty} \frac{d z}{(z+x+1)^{1-2 \beta}(z+y+1)^{1+2 \beta}} .
\end{aligned}
$$

Now we remark that $K_{\alpha}$ also depends on $\beta$, but we suppress this dependence since we are eventually going to think of $D_{n}[\sigma]$ as a function of the $\alpha_{r}$ 's. In [16] it is shown that $I-K_{\alpha}$ is invertible for $|R \alpha|<\frac{1}{2}$ and $f=\left(I-K_{\alpha}\right)^{-1} g$ is given by the following.

Let

$$
G(s)=\int_{0}^{\infty}(x+1)^{s-1} g(x) d x, \quad R s<\frac{1}{2} .
$$

The function $G(s)$ belongs to $H_{2}$ of the half-plane $R s<\frac{1}{2}$. Therefore it has a boundary function that exists a.e. and belongs to $L_{2}$ of $R s=\frac{1}{2}$. Thus the function

$$
\frac{\Gamma(t-\alpha+\beta) \Gamma(t+\alpha+\beta)}{\Gamma(t) \Gamma(t+2 \beta)} \frac{G(t)}{(t-s)}
$$

is in $L_{1}$ of $R t=\frac{1}{2}$ for $R s<\frac{1}{2}$ and we can define a function $G_{-}(s)$ by

$$
G_{-}(s)=\frac{1}{2 \pi i} \int_{1 / 2-i \infty}^{1 / 2+i \infty} \frac{\Gamma(t-\alpha+\beta) \Gamma(t+\alpha+\beta) G(t)}{\Gamma(t) \Gamma(t+2 \beta)(t-s)} d t
$$

$$
R s<\frac{1}{2}
$$

Then if

$$
F_{-}(s)=\int_{1}^{\infty} x^{s-1} f(x-1) d x
$$

we have

$$
F_{-}(s)=\frac{\Gamma(1-s-\alpha-\beta) \Gamma(1-s+\alpha-\beta)}{\Gamma(1-s) \Gamma(1-2 \beta-s)} G_{-}(s)
$$

and

$$
f(x)=\frac{1}{2 \pi i} \int_{1 / 2-i \infty}^{1 / 2+i \infty} F_{-}(s)(x+1)^{-s} d s
$$

The operator $\Sigma_{r}\left(I-K_{\alpha_{r}}\right)^{-1}$ will be used to approximate $(I-U V)^{-1}$ and this will lead to an approximation for the $\psi(z)$ in Proposition 2.3. Recall $U V$ was the operator on $z H_{2} \rightarrow z H_{2}$ that sent $\phi \rightarrow\left(z^{-n}\left(z^{n} \phi \sigma_{+} / \sigma_{-}\right)_{-} \sigma_{-} / \sigma_{+}\right)_{+}$. Let us look at the functions $\sigma_{+} / \sigma_{-}$and $\sigma_{-} / \sigma_{+}$. From (1.6) and (1.7) the functions $\sigma_{+}$and $\sigma_{-}$are given by 


$$
\begin{aligned}
& \sigma_{+}(z)=\tau_{+}(z) \prod_{r=1}^{R}\left(1-\frac{z}{z_{r}}\right)^{\alpha_{r}+\beta_{r}}, \\
& \sigma_{-}(z)=\tau_{-}(z) \prod_{r=1}^{R}\left(1-\frac{z_{r}}{z}\right)^{\alpha_{r}-\beta_{r}},
\end{aligned}
$$

where $\tau_{+}$and $\tau_{-}$have 1 as the constant terms of their Fourier expansion and these functions have derivatives satisfying Lipschitz conditions. Notice in the above formulas we have used $\tau(z)$ instead of $\tau(\theta)$. Here $\tau(z)=\tau(\theta)$ where $z=e^{i \theta}$. This will be used whenever convenient. It follows that for $k>0$, $k \rightarrow \infty$,

$$
\begin{aligned}
& \frac{1}{2 \pi} \int_{-\pi}^{\pi} \frac{\sigma_{+}}{\sigma_{-}} e^{i k \theta} d \theta \\
& \quad=\frac{1}{\pi} \sum_{r=1}^{R} a_{r} z_{r}^{k} \frac{\Gamma\left(1+2 \beta_{r}\right) \sin \pi\left(\alpha_{r}-\beta_{r}\right)}{k^{1+2 \beta_{r}}}+O\left(k^{-1-\delta}\right),
\end{aligned}
$$

$$
\begin{aligned}
& \frac{1}{2 \pi} \int_{-\pi}^{\pi} \frac{\sigma_{-}}{\sigma_{+}} e^{-i k \theta} d \theta \\
& \quad=\frac{1}{\pi} \sum_{r=1}^{R} b_{r} z_{r}^{-k} \frac{\Gamma\left(1-2 \beta_{r}\right) \sin \pi\left(\alpha_{r}+\beta_{r}\right)}{k^{1-2 \beta_{r}}}+O\left(k^{-1-\delta}\right)
\end{aligned}
$$

where

$$
\begin{aligned}
& a_{r}=\frac{\tau_{+}}{\tau_{-}}\left(z_{r}\right) \prod_{s \neq r}\left(-e^{i\left(\theta_{r}-\theta_{s}\right)}\right)^{\alpha_{r}}\left(2-2 \cos \left(\theta_{r}-\theta_{s}\right)\right)^{\beta_{r}}, \\
& b_{r}=\frac{\tau_{-}}{\tau_{+}}\left(z_{r}\right) \prod_{s \neq r}\left(-e^{i\left(\theta_{r}-\theta_{s}\right)}\right)^{-\alpha_{r}}\left(2-2 \cos \left(\theta_{r}-\theta_{s}\right)\right)^{-\beta_{r}}
\end{aligned}
$$

and $a_{r} \cdot b_{r}=1$.

Now $z^{k}(k \geqslant 1)$ is a basis for $z H_{2}$. So consider $U V$ as a semi-infinite matrix. Using (2.8) and (2.9) we see that $U V$ has $j, k$ entry

$$
\begin{gathered}
\pi^{-2} \sum_{l=1}^{\infty}\left\{\sum_{r=1}^{R} b_{r} z_{r}^{-(l+j+n)} \frac{\Gamma\left(1-2 \beta_{r}\right) \sin \pi\left(\alpha_{r}+\beta_{r}\right)}{(l+j+n)^{1-2 \beta_{r}}}+O\left((l+j+n)^{-1-\delta}\right)\right\} \\
\times\left\{\sum_{s=1}^{R} a_{s} z_{s}^{(l+k+n)} \frac{\Gamma\left(1+2 \beta_{s}\right) \sin \pi\left(\alpha_{s}-\beta_{s}\right)}{(l+k+n)^{1+2 \beta_{s}}}\right. \\
\left.+O\left((l+k+n)^{-1-\delta}\right)\right\} .
\end{gathered}
$$

When multiplying these expressions the contribution from terms involving $O$ 's is $O\left((j+n)^{-1 / 2-\delta}(k+n)^{-1 / 2-\delta}\right)$.

If $r \neq s$ we have a sum of the form 


$$
\sum_{l=1}^{\infty} \frac{\left(z_{s} / z_{r}\right)^{l}}{(l+j+n)^{1-2 \beta_{r}}(l+k+n)^{1+2 \beta_{s}}} .
$$

If we sum this by parts, using the fact that the partial sums of $\Sigma_{l}\left(z_{s} / z_{r}\right)^{l}$ are bounded, and apply the mean value theorem to the terms of

$$
\begin{aligned}
\sum_{l=1}^{\infty}\left[\frac{1}{(l+j+n)^{1-2 \beta_{r}}(l+k+n)^{1+2 \beta_{s}}}\right. & \\
& \left.\quad-\frac{1}{(l+1+j+n)^{1-2 \beta_{r}}(l+1+k+n)^{1+2 \beta_{s}}}\right],
\end{aligned}
$$

we see that this sum is $O\left((j+n)^{-1}(k+n)^{-1}\right)$. We are left with

$$
\begin{aligned}
\pi^{-2} \sum_{r=1}^{R}\left\{z_{r}^{k-j}\right. & \Gamma\left(1-2 \beta_{r}\right) \Gamma\left(1+2 \beta_{r}\right) \sin \pi\left(\alpha_{r}+\beta_{r}\right) \\
& \left.\times \sin \pi\left(\alpha_{r}-\beta_{r}\right) \sum_{l=1}^{\infty}(l+j+n)^{-1+2 \beta_{r}}(l+k+n)^{-1-2 \beta_{r}}\right\} \\
& +O\left((j+n)^{-1 / 2-\delta}(k+n)^{-1 / 2-\delta}\right) .
\end{aligned}
$$

Now let us jump another step and think of the semi-infinite matrix $U V$ as acting on a subspace of $L_{2}(0, \infty)$ consisting of functions which are constant on $(k-1, k]$. Let $\{x\}=$ the smallest integer not less than $x$. Then $U V$ may be thought of as the integral operator with kernel

$$
\begin{aligned}
& \pi^{-2} \sum_{r=1}^{R}\left\{z_{r}^{\{y\}-\{x\}} \Gamma\left(1-2 \beta_{r}\right) \Gamma\left(1+2 \beta_{r}\right) \sin \pi\left(\alpha_{r}-\beta_{r}\right)\right. \\
& \left.\quad \times \sin \pi\left(\alpha_{r}+\beta_{r}\right) \sum_{l=1}^{\infty}(l+\{x\}+n)^{-1+2 \beta_{r}}(l+\{y\}+n)^{-1-2 \beta_{r}}\right\} \\
& +O\left((\{x\}+n)^{-1 / 2-\delta}(\{y\}+n)^{-1 / 2-\delta}\right) \\
& =\pi^{-2} \sum_{r=1}^{R}\left\{z_{r}^{(y)-\{x\}} \Gamma\left(1-2 \beta_{r}\right) \Gamma\left(1+2 \beta_{r}\right) \sin \pi\left(\alpha_{r}-\beta_{r}\right)\right. \\
& \left.\quad \times \sin \pi\left(\alpha_{r}+\beta_{r}\right) \int_{0}^{\infty} \frac{d z}{(z+x+n)^{1-2 \beta_{r}}(z+y+n)^{1+2 \beta_{r}}}\right\} \\
& +O\left((x+n)^{-1 / 2-\delta}(y+n)^{-1 / 2-\delta}\right) \\
& =\sum_{r=1}^{R} K_{r}+K_{e}=\sum_{i=1}^{R+1} K_{i} \quad\left(K_{R+1}=K_{e}\right) .
\end{aligned}
$$


LEMMA 2.4.The operator $I-U V=I-\sum_{r=1}^{R} K_{r}-K_{e}$ is invertible.

Since

$$
K_{r} f(x)=\left[z_{r}^{-\{n x\}} K_{\alpha_{r}}\left(z_{r}^{\{n x\}} f(n x)\right)\right](x / n),
$$

each $I-K_{r}$ is invertible (since $I-K_{\alpha_{r}}$ is invertible). The operator $I-K_{e}$ is invertible also for sufficiently large $n$ since $K_{e}$ has norm $O\left(n^{-\delta}\right)$, where this $O$ refers to the $L_{2}$ norm of the operator $K_{e}$. Consider the following identity:

$$
\left\{\sum_{i=1}^{R+1}\left(I-K_{i}\right)^{-1}-R I\right\}\left\{I-\sum_{i=1}^{R+1} K_{i}\right\}=I+\sum_{i \neq i^{\prime}}\left(I-K_{i}\right)^{-1} K_{i} K_{i^{\prime}}
$$

which can be derived from the identity

$$
(I-K)^{-1}=I+(I-K)^{-1} K \text {. }
$$

If $i \neq i^{\prime}<R+1$, integration by parts shows $K_{i} K_{i^{\prime}}$ has kernel

$$
\begin{aligned}
O\left(\int_{0}^{\infty}\right. & \frac{d z}{(z+x+n)^{1-2 \beta_{r}}(z+n)^{1+2 \beta_{r}}} \\
& \left.\times \int_{0}^{\infty} \frac{d z}{(z+n)^{1-2 \beta_{r}}(z+y+n)^{1+2 \beta_{r}}}\right) .
\end{aligned}
$$

From this last expression it follows $K_{i} K_{i^{\prime}}$ has norm $O\left(n^{-1}\right)$. The operator $K_{e}$ has norm $O\left(n^{-\delta}\right)$ and so we can say the right side of $(2.10)$ is $I+O\left(n^{-\delta}\right)$. It follows that

$$
\begin{aligned}
\left(I-\sum_{r=1}^{R+1} K_{i}\right)^{-1} & =\sum_{i=1}^{R+1}\left(I-K_{r}\right)^{-1}-R I+O\left(n^{-\delta}\right) \\
& =\sum_{i=1}^{R}\left(I-K_{r}\right)^{-1}-(R-1) I+O\left(n^{-\delta}\right) .
\end{aligned}
$$

Now

$$
I-U V=I-\sum_{r=1}^{R+1} K_{i}
$$

Hence $I-U V$ is invertible for sufficiently large $n$. The same statement is valid for $I-V U$, and we can conclude from Proposition 2.1 that $T_{n}[\sigma]^{-1}$ exists for the same $n$.

We have now proved the first part of Proposition 2.3 and we turn now to the estimate for the integral

$$
\frac{1}{2 \pi} \int_{-\pi}^{\pi} z^{n} \psi(z) \frac{\sigma_{+}(z)}{\sigma_{-}(z)} d \theta
$$


The function $\psi(z)$ here is from (2.3):

$$
(I-U V)^{-1}\left[-\left(z^{-n}\left(\sigma_{-} / \sigma_{+}\right)\left(q / \sigma_{-}\right)^{+}\right)_{+}\right] \text {, }
$$

where $q=1$. We proceed to solve this now for a general $q$. Although this is not necessary in this section, it will be helpful later on and take care of $q=1$ at the same time.

So let $q=z^{i}$. Then

$$
-\left(z^{-n} \frac{\sigma_{-}}{\sigma_{+}}\left(\frac{q}{\sigma_{-}}\right)^{+}\right)_{+}=-\left(\frac{\sigma_{-}}{\sigma_{+}} \sum_{m=n-i}^{n}\left(\frac{1}{\sigma_{-}}\right)_{n-m-i} z^{-m}\right)_{+} .
$$

Call $\psi^{m}$ the solution to

$$
(I-U V) \psi=\left(z^{-m_{-}} \sigma_{-} / \sigma_{+}\right)_{+} \cdot
$$

From the above computation one can see that a linear combination on the $\psi^{m}$ yields a solution to $\psi(z)$ corresponding to $q=z^{i}$ and, thus, eventually to any polynomial. Notice that in the special case $q=1=z^{0}$ we have $\psi(z)=$ $-\psi^{n}(z)$

We now prove

\section{LEMMA 2.5.}

$$
\begin{aligned}
\psi_{\{x\}}^{m}=\pi^{-1} \sum_{r=1}^{R}\left[b_{r} z_{r}^{-\{x\}-m} \Gamma\left(1-2 \beta_{r}\right) \sin \pi\left(\alpha_{r}+\beta_{r}\right)\right. \\
\left.\quad \times\left(I-K_{\alpha_{r}}\right)^{-1}(n x+m+1)^{-1+2 \beta_{r}}(x / n)\right] \\
+O\left((m+1)^{-1 / 2-\delta}\right),
\end{aligned}
$$

and this $O$ refers to the $L_{2}$ norm.

To prove this let us return to the infinite matrix situation where

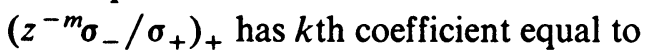

$$
\begin{aligned}
\frac{1}{\pi} \sum_{r=1}^{R} b_{r} z_{r}^{-k-m} & \frac{\Gamma\left(1-2 \beta_{r}\right) \sin \pi\left(\alpha_{r}+\beta_{r}\right)}{(k+m+1)^{1-2 \beta_{r}}} \\
& +O\left((k+m+1)^{-1-\delta}\right) \\
= & \frac{1}{\pi} \sum_{r=1}^{R} b_{r} z_{r}^{-(x)-m} \frac{\Gamma\left(1-2 \beta_{r}\right) \sin \pi\left(\alpha_{r}+\beta_{r}\right)}{(x+m+1)^{1-2 \beta_{r}}} \\
& +O\left((x+m+1)^{-1-\delta}\right) \\
= & \sum_{1}^{R} g_{r}+g_{e}
\end{aligned}
$$


and

$$
\left\|g_{e}\right\|_{2}=O\left((m+1)^{-1 / 2-\delta}\right) .
$$

So for $\psi_{\{x\}}^{m}$ we have

$$
\begin{aligned}
\psi_{(x)}^{m}= & (I-U V)^{-1}\left(\sum_{1}^{R} g_{r}+g_{e}\right) \\
= & {\left[\sum_{r=1}^{R}\left(I-K_{r}\right)^{-1}-(R-1) I+O\left(n^{-\delta}\right)\right]\left(\sum_{1}^{R} g_{r}+g_{e}\right) } \\
= & {\left[\sum_{r=1}^{R}\left(I-K_{r}\right)^{-1}\right]\left(\sum_{1}^{R} g_{s}+g_{e}\right)-(R-1)\left(\sum_{1}^{R} g_{r}+g_{e}\right) } \\
& +O\left(n^{-\delta}(m+1)^{-1 / 2}\right)
\end{aligned}
$$

since the norm of each $g_{r}$ is at most $(m+1)^{-1 / 2}$. From (2.10),

$$
\left(I-K_{r}\right)^{-1} g_{s}=\left(I-K_{r}\right)^{-1} K_{r} g_{s}+g_{s} \text {. }
$$

If $r \neq s$, integration by parts shows that

$$
K_{r} g_{s}=O\left(\int_{0}^{\infty} \frac{d z}{(z+x+n)^{1+2 \beta_{r}}(m+1)^{1-2 \beta_{r}}(z+m)^{1-2 \beta_{r}}}\right)
$$

which has $L_{2}$ norm $O\left((m+1)^{-1} n^{-1 / 2}\right)$. So $\psi_{\{x\}}^{m}$ becomes

$$
\begin{aligned}
\sum_{r=1}^{R}(I- & \left.K_{r}\right)^{-1} g_{r}+\sum_{r \neq s}\left(I-K_{r}\right)^{-1} g_{s} \\
& +\sum_{r=1}^{R}\left\{\left(I-K_{r}\right)^{-1} g_{e}-(R-1)\left(g_{r}+g_{e}\right)\right\}+O\left(n^{-\delta}(m+1)^{-1 / 2}\right) \\
= & \sum_{r=1}^{R}\left(I-K_{r}\right)^{-1} g_{r}+\sum_{r \neq s}\left(\left(I-K_{r}\right)^{-1} K_{r} g_{s}+g_{s}\right) \\
& +\sum_{r=1}^{R}\left\{\left(I-K_{r}\right)^{-1} g_{e}-(R-1)\left(g_{r}+g_{e}\right)\right\}+O\left(n^{-\delta}(m+1)^{-1 / 2}\right) \\
= & \sum_{r=1}^{R}\left(I-K_{r}\right)^{-1} g_{r}+\sum_{r=1}^{R}(R-1) g_{r}+O\left(n^{-1 / 2}(m+1)^{-1}\right) \\
& +\sum_{r=1}^{R}\left[\left(I-K_{r}\right)^{-1} g_{e}-(R-1)\left(g_{r}+g_{e}\right)\right]+O\left(n^{-\delta}(m+1)^{-1 / 2}\right) \\
= & \sum_{r=1}^{R}\left(I-K_{r}\right)^{-1} g_{r}+O\left((m+1)^{-1 / 2-\delta}\right) .
\end{aligned}
$$


Thus we can say

$$
\begin{aligned}
\psi_{\{x\}}^{m}=\pi^{-1} \sum_{r=1}^{R}\left[b_{r} z_{r}^{-\{x\}-m} \Gamma\left(1-2 \beta_{r}\right) \sin \pi\left(\alpha_{r}+\beta_{r}\right)\right. \\
\left.\quad \times\left(I-K_{\alpha_{r}}\right)^{-1}(n x+m+1)^{-1+2 \beta_{r}}(x / n)\right] \\
+O\left((m+1)^{-1 / 2-\delta}\right) .
\end{aligned}
$$

We now complete the proof of Proposition 2.4. By Lemma 2.5 for $q=1$, we have

$$
\begin{aligned}
& \psi_{\{x\}=}=-\psi^{n}\{x\} \\
&=-\pi^{-1} \sum_{r=1}^{R}\left[b_{r} z_{r}^{-\{x\}-n} \Gamma\left(1-2 \beta_{r}\right) \sin \pi\left(\alpha_{r}+\beta_{r}\right)\right. \\
&\left.\quad \quad \times\left(I-K_{\alpha_{r}}\right)^{-1}(n x+n)^{-1+2 \beta_{r}}(x / n)\right]+O\left(n^{-1 / 2}\right) .
\end{aligned}
$$

The function $\psi_{\{x\}}$ will mean this special one until the end of this section. Our final step in the proof is to write the integral

$$
\frac{1}{2 \pi} \int_{-\pi}^{\pi} z^{n} \psi(z) \frac{\sigma_{+}(z)}{\sigma_{-}(z)} d \theta \text { as } \int_{0}^{\infty} \psi_{\{x\}} \zeta_{\{x\}+n} d x,
$$

where $\zeta_{\{x\}+n}$ is the $-\{x\}-n$th Fourier coefficient of $z^{n} \sigma_{+}(z) / \sigma_{-}(z)$, and do some substitution. We already have an expression for $\psi_{\{x\}}$, namely (2.12), and from (2.8) one can see that

$$
\zeta_{\{x\}+n}=\frac{1}{\pi} \sum_{r=1}^{R} a_{r} z_{r}^{\{x\}+n} \frac{\Gamma\left(1+2 \beta_{r}\right) \sin \pi\left(\alpha_{r}-\beta_{r}\right)}{(x+n)^{1+2 \beta_{r}}}+O\left((x+n)^{-1-\delta}\right) .
$$

When one multiplies $\psi_{\{x\}}^{m}$ and $\zeta_{\{x\}+n}$ and integrates from 0 to $\infty$, the cross $O$ terms contribute $O\left(n^{-1-\delta}\right)$. The remaining terms are a double sum $\Sigma_{r, s} \int \ldots d x$. Let $r \neq s$. Then we are integrating a constant times

$$
\left(\frac{z_{s}}{z_{r}}\right)^{\{x\}}\left[\left(1-K_{\alpha_{r}}\right)^{-1}(n x+n)^{-1+2 \beta_{r}}\left(\frac{x}{n}\right)\right](x+n)^{-1-2 \beta_{r}}
$$

Integration by parts shows that this is $n^{-2}$ times the total variation of $\left[\left(I-K_{\alpha_{r}}\right)^{-1}(x+1)^{-1+2 \beta_{r}}\right](x / n)$. Let

$$
\left(I-K_{\alpha_{r}}\right)^{-1}(x+1)^{-1+2 \beta_{r}}=f_{\alpha_{r}} .
$$

Then

$$
\left(I-K_{\alpha_{r}}\right) f_{\alpha_{r}}=(x+1)^{-1+2 \beta_{r}}
$$

or 


$$
f_{\alpha_{r}}=(x+1)^{-1+2 \beta_{r}}+K_{\alpha_{r}} f_{\alpha_{r}} .
$$

The variation of $K_{\alpha_{1}} f_{\alpha_{1}}$ equals $O\left(\|(x+1)^{-1+2 \beta_{r} \|_{2}}\right)$ which is $O(1)$, and the variation of $(x+1)^{-1+2 \beta_{r}}$ is also finite. Hence the variation of $\left(I-K_{\alpha}\right)^{-1}(x$ $+1)^{-1+2 \beta_{r}}$ is finite and these cross terms contribute at most $O\left(n^{-2}\right)$. Therefore the integral in Proposition 2.3 is equal to

$$
-\pi^{-2} \sum_{r=1}^{R} \Gamma\left(1+2 \beta_{r}\right) \Gamma\left(1-2 \beta_{r}\right) \sin \pi\left(\alpha_{r}-\beta_{r}\right) \sin \pi\left(\alpha_{r}+\beta_{r}\right)
$$

$$
\begin{aligned}
& \times \int_{0}^{\infty}\left[\left(I-K_{\alpha_{r}}\right)^{-1}(n x+n)^{-1+2 \beta_{r}}\left(\frac{x}{n}\right)\right](x+n)^{-1-2 \beta_{r}} d x \\
& +O\left(n^{-1-\delta}\right) .
\end{aligned}
$$

Using (2.5)-(2.7) we can compute the remaining integral. We need to evaluate $F_{-}(-2 \beta)$ where, $g(x)=(x+1)^{-1+2 \beta}$ (using the notation for formulas (2.5)-(2.7)). For this $g(x)$ we have

$$
\begin{aligned}
G(s) & =(1-s-2 \beta)^{-1} \\
G_{-}(s) & =\frac{\Gamma(1-\alpha-\beta) \Gamma(1+\alpha-\beta)}{\Gamma(1-2 \beta)}(1-2 \beta-s)^{-1} .
\end{aligned}
$$

So

$$
\begin{aligned}
F_{-}(-2 \beta) & =\frac{\Gamma(1+\beta-\alpha) \Gamma(1+\beta+\alpha) \Gamma(1-\alpha-\beta) \Gamma(1+\alpha-\beta)}{\Gamma(1-2 \beta) \Gamma(1+2 \beta)} \\
& =\left(\alpha^{2}-\beta^{2}\right) \pi^{2} \frac{\csc \pi(\alpha-\beta) \csc \pi(\alpha+\beta)}{\Gamma(1+2 \beta) \Gamma(1-2 \beta)} .
\end{aligned}
$$

Substituting this in (2.13) we have

$$
\int_{0}^{\infty} \psi_{\{x\}} \zeta_{\{x\}+n}=-\sum\left(\alpha_{r}^{2}-\beta_{r}^{2}\right) n^{-1}+O\left(n^{-1-\delta}\right) .
$$

Thus Proposition 2.3 has been proved and (2.1) is valid.

From the existence of the limit of $S_{n}[\sigma]$ we can say that for fixed $\alpha_{r}$ in $\left|R \alpha_{r}\right|<\frac{1}{2}$ we have

$$
S_{n}[\sigma]=O(1) .
$$

In $\S 5$, we will need to know that (2.14) holds uniformly for $\left|R \alpha_{r}\right|<\frac{1}{2}$ and $\left|\alpha_{r}\right|$ bounded. To see this notice that if we move the line of integration to the left in (2.7) we have an estimate $\left\|(1+x)^{-\eta}\left(I-K_{\alpha}\right)^{-1} g\right\|_{2}=O\left(\|g\|_{2}\right)$ for each $\eta>0$. This last estimate is uniform for $\left|R \alpha_{r}\right|<\frac{1}{2}$ and $\left|\alpha_{r}\right|$ bounded. Using this estimate we can use the methods of $[16, \S \mathrm{VI}]$ and say that $(2.14)$ holds uniformly for $\left|R \alpha_{r}\right|<\frac{1}{2}$ and $\left|\alpha_{r}\right|$ bounded. 
3. Preliminary form of the asymptotic formula for $D_{n}[\sigma]$. The last section was concerned with the existence of the limit of $S_{n}[\sigma]$ for $\left|R_{\alpha}\right|<\frac{1}{2}$. $\$ 3$ will be concerned with finding a preliminary form of the asymptotic formula for $D_{n}[\sigma]$. We begin by stating the following result found in [9]. If $p=T_{n}[\sigma]^{-1} 1$ and $q=T_{n}[\overline{\sigma(\theta)}]^{-1} 1$, and if $p$ and $q$ have zeros only outside the unit circle, then

$$
D_{n}[\sigma]=\left(D_{n}[\sigma] / D_{n-1}[\sigma]\right)^{n+1} E(n)
$$

where

$$
E(n)=\exp \left\{-\frac{1}{2 \pi i} \int_{-\pi}^{\pi} \log p\left(e^{i \theta}\right) d \log \overline{q\left(l^{i \theta}\right)}\right\} .
$$

This indicates that we need more information about $p=T_{n}[\sigma]^{-1} 1$. We make in this section, for computational purposes, the assumption that each $\alpha_{r}$ is real and $\left|\alpha_{r}\right|<\frac{1}{2}$. From Proposition 2.1 the equation for $p$ is

$$
p=1 / \sigma+\phi / \sigma_{+}+z^{n} \psi / \sigma_{-}
$$

or

$$
\begin{gathered}
p \sigma_{+}=1 / \sigma_{-}+\phi+z^{n} \psi \sigma_{+} / \sigma_{-}, \\
1 / \sigma_{-} \in \bar{H}_{2}, \quad \phi \in \overline{z H}_{2}, \quad p \sigma_{+} \in H_{2} .
\end{gathered}
$$

So

$$
p \sigma_{+}=1+\left(z^{n} \psi \sigma_{+} / \sigma_{-}\right)^{+} .
$$

Using the expression obtained in Lemma 2.5 for $\psi_{\{x\}}$ we can write $\left(z^{n} \psi \sigma_{+} / \sigma_{-}\right)^{+}$as

$$
\begin{aligned}
& -\pi^{-1} \sum_{r=1}^{R} n^{-1+2 \beta_{r}} b_{r} \sin \pi\left(\alpha_{r}+\beta_{r}\right) \Gamma\left(1-2 \beta_{r}\right) z_{r}^{-n} \\
& \quad \times\left(\frac{\sigma_{+}}{\sigma_{-}} \sum_{k=-\infty}^{\infty} f_{\alpha_{r}}\left(\frac{k}{n}\right) z_{r}^{-k} z^{n+k}\right)^{+}+O\left(n^{-1 / 2-\delta}\right),
\end{aligned}
$$

where

$$
f_{\alpha_{r}}(x)=\left[\left(I-K_{\alpha_{r}}\right)^{-1}(x+1)^{-1+2 \beta_{r}}\right](x)
$$

and $f_{\alpha,}$ is defined to be zero for $x<0$. We can now follow essentially the same procedure used in [16] and replace several terms in this last expression with approximations that lead to something that one can actually compute. The reader is referred to [16, IX, pp. 358-362]. We only mention here that the essential change is to replace $\left(\sigma_{+} / \sigma_{-}\right)\left(e^{i x / n}\right)$ by $a_{r} e^{i \pi \alpha_{s} g n x}(x / n)^{2 \beta_{r}}$ for $x>0$,

$$
a_{r} e^{i \pi \alpha_{,} \operatorname{sgn} x}(-x / n)^{2 \beta_{r}} \text { for } x<0
$$

instead of the replacement used in [16, IX]. This produces the following 
results, which are analogous to those found in [16, IX, p. 367].

We first set

$$
\Psi_{\alpha}(\theta)=\frac{\Gamma(\alpha-\beta+1)}{\Gamma(2 \alpha+1)} e^{-i \pi(\alpha+\beta) / 2} \theta^{\alpha+\beta} \Phi(\alpha+\beta, 2 \alpha+1,-i \theta),
$$

where $\Phi$ is the confluent hypergeometric function with parameters $\alpha+\beta$ and $2 \alpha+1[2,6.1,(1)]$, and where we take $0<\arg \theta<\pi$ for $9 \theta>0$. Now suppose we let

$$
\lambda(\theta)=\Pi\left(1-e^{i\left(\theta-\theta_{r}\right)}\right)^{\alpha_{r}+\beta_{r}} / \Psi_{\alpha_{r}}\left(n\left(\theta-\theta_{r}\right)\right) .
$$

Then one has the following estimates:

$$
\begin{gathered}
\left\|p\left(e^{i \theta}\right) \tau_{+}\left(e^{i \theta}\right) \lambda\left(e^{i \theta}\right)-1\right\|_{\infty}=O\left(n^{-\delta}\right), \\
\left\|\log p\left(e^{i \theta}\right) \tau_{+}\left(e^{i \theta}\right) \lambda(\theta)\right\|_{\infty}=O\left(n^{-\delta}\right), \\
\left\|\log p\left(e^{i \theta}\right) \tau_{+}\left(e^{i \theta}\right) \lambda(\theta)\right\|_{2}=O\left(n^{-1 / 2-\delta}\right), \\
\left\|\left\{\log p\left(e^{i \theta}\right) \tau_{+}\left(e^{i \theta}\right) \lambda(\theta)\right\}^{\prime}\right\|_{2}=O\left(n^{1 / 2-\delta}\right) .
\end{gathered}
$$

Here we choose the principal branch of the logarithm, and these norms are all taken over the interval $I$, where $I$ is chosen to be any closed interval of length $2 \pi$ which contains for each $r$ a $\theta_{r}$ satisfying $z_{r}=e^{i \theta_{r}}$.

If $\sigma$ is nonnegative,

$$
p\left(e^{i \theta}\right)=e^{i n \theta} \phi_{n}\left(e^{-i \theta}\right),
$$

where $\phi_{n}$ is the suitably normalized $n$th orthonogonal polynomial associated with $\sigma(-\theta)[13,11.1]$. Thus the above formulas give the uniform asymptotic formulas for orthogonal polynomials promised in the introduction.

We should also remark here that the introduction of confluent hypergeometric functions is not surprising. The analogous results of Widom contain expressions involving Bessel functions which can be written in terms of confluent hypergeometric functions. However in this more general case the function $\Psi_{\alpha}(\theta)$ cannot be reduced to an expression containing Bessel functions.

The estimates just obtained are useful because they can be substituted into (3.1). We can duplicate the analysis of [16] and write $\log E(n)$ as

$$
-\frac{1}{2 \pi i} \int_{I} f_{0}(\theta) d g_{0}(\theta)+O\left(n^{-\delta}\right)
$$

where

$$
f_{0}(\theta)=\log \tau_{+}\left(e^{i \theta}\right) \lambda(\theta), \quad g_{0}(\theta)=\log \tau_{-}\left(e^{i \theta}\right) \overline{\lambda(\theta)} .
$$

See [16, X, pp. 367-368]. 
The above integral can then be written as a sum of integrals in an obvious way. Using integration by parts several times and the fact that

$$
\left\|\log \prod_{r} \Psi_{\alpha_{r}}\left(n\left(\theta-\theta_{r}\right)\right)\right\|_{1}=O(\log n / n)
$$

(see $[2,6.13 .1,(3)]$ and $[16, X$, pp. 368-370]), we are left with the following formula:

$$
\begin{aligned}
\log E(n)= & \log E[\tau]-\sum_{r}\left(\alpha_{r}+\beta_{r}\right) \log \tau_{-}\left(z_{r}\right) \\
& -\sum_{r}\left(\alpha_{r}-\beta_{r}\right) \log \tau_{+}\left(z_{r}\right) \\
& -\sum_{r \neq s}\left(\alpha_{r}+\beta_{r}\right)\left(\alpha_{s}-\beta_{s}\right) \log \left(1-e^{i\left(\theta_{s}-\theta_{r}\right)}\right) \\
& +\sum_{r} F\left(n, \alpha_{r}, \beta_{r}\right)+O\left(n^{-\delta}\right),
\end{aligned}
$$

where

$$
F(n, \alpha, \beta)=-\frac{1}{2 \pi i} \int_{-\pi}^{\pi} \log \frac{\left(1-e^{i \theta}\right)^{\alpha+\beta}}{\Psi_{\alpha}(n \theta)} d \log \frac{\left(1-e^{-i \theta}\right)^{\alpha-\beta}}{\overline{\Psi_{\alpha}(n \theta)}} d \theta
$$

Now let us return to (3.1). The factor $\left(D_{n}[\sigma] / D_{n-1}[\sigma]\right)^{n+1}$ can be written as $\exp \left\{\Sigma\left(\alpha_{r}^{2}-\beta_{r}^{2}\right)+O\left(n^{-\delta}\right)\right\}$. This can be seen from (2.1). We have just determined the factor $E(n)$ except for the terms $F\left(n, \alpha_{r}, \beta_{r}\right)$. These last terms depend on $n, \alpha_{r}$ and $\beta_{r}$. Therefore, using (2.1) we will be able to determine an asymptotic formula for $D_{n}[\sigma]$ (at least when $\left|R \alpha_{r}\right|<\frac{1}{2}$ and $9 \alpha_{r}=0$ ) if we can asymptotically evaluate $F(n, \alpha, \beta)$. We have therefore reduced the problem to finding an asymptotic formula for $D_{n}[\sigma]$ where

$$
\sigma(\theta)=\left(1-e^{i \theta}\right)^{\alpha+\beta}\left(1-e^{i \theta}\right)^{\alpha-\beta} .
$$

We will do this in the last two sections of the paper.

4. A difference equation. In this section we let $D_{n}[\alpha]$ denote $D_{n}[\sigma]$ where $\sigma(\theta)=\left(1-e^{i \theta}\right)^{\alpha+\beta}\left(1-e^{-i \theta}\right)^{\alpha-\beta}$. Our first step in finding an asymptotic formula for $D_{n}[\alpha]$ is the following.

Proposition 4.1. For $|R \alpha|<\frac{1}{2}$,

$$
\lim _{n \rightarrow \infty} \frac{D_{n-1}[\alpha+1]}{D_{n}[\alpha]} n^{-2 \alpha-1}=\frac{\Gamma(1+\alpha+\beta) \Gamma(1+\alpha-\beta)}{\Gamma(1+2 \alpha) \Gamma(2+2 \alpha)} .
$$

We begin the proof by stating the following lemma, which appears in $[16$, §VII]. If $T_{n}[\sigma]$ is invertible then 


$$
\begin{aligned}
& D_{n-p}\left[\sigma \prod_{r=1}^{p}\left(1-\frac{z}{z_{r}}\right)\left(1-\frac{z_{r}}{z}\right)\right] \\
& \quad=\prod_{1<r<s<p}\left|z_{r}-z_{s}\right|^{-2} D_{n}[\sigma]\left|T_{n}[\sigma]^{-1}\left(\sum_{s=0}^{n} z_{s}^{-k} z^{k}\right)\left(z_{r}\right)\right|_{r, s=1, \ldots, p .}
\end{aligned}
$$

This lemma indicates that as a function of $\alpha, D_{n}[\alpha]$ should satisfy a difference equation. In fact for $p=1, z_{r}=1, \sigma=\left(1-e^{i \theta}\right)^{\alpha+\beta}\left(1-e^{-i \theta}\right)^{\alpha-\beta}$, we have

$$
D_{n-1}[\alpha+1]=D_{n}[\alpha]\left\{\left[T_{n}[\alpha]^{-1}\left(\sum_{s=0}^{n} z^{k}\right)\right](1)\right\} \text {. }
$$

We proceed now to determine for $|R \alpha|<\frac{1}{2}$ the second factor of the righthand side of this equation. From Proposition 2.1, the polynomial $p=$ $T_{n}[\alpha]^{-1} q$ is given by

$$
p=q / \sigma+\phi / \sigma_{+}+z^{n} \psi / \sigma_{-}
$$

or

$$
p_{[0, n]}(1)=\left(\frac{q}{\sigma}\right)_{[0, n]}(1)+\left(\frac{\phi}{\sigma_{+}}\right)_{[0, n]}(1)+\left(\frac{z^{n} \psi}{\sigma_{-}}\right)_{[0, n]}(1) .
$$

Here $f_{[a, b]}$ means $\sum_{k=a}^{b} f_{k} z^{k}$. Each of these terms must be estimated at $z=1$. Let us restrict ourselves first to $z^{n}\left(\psi / \sigma_{-}\right)_{[0, n]}(1)$. The function $\psi$ in this case corresponds to $q=\sum_{0}^{n} z^{k}$. From (2.11) $\psi$ is given by

$$
-\sum_{i=0}^{n}\left(\sum_{m=n-i}^{n}\left(\left(\frac{1}{\sigma_{-}}\right)_{n-m-i}\right) \psi^{m}\right)=-\sum_{m=0}^{n} s_{m}\left(\frac{1}{\sigma_{-}}\right) \psi^{m}
$$

where $s_{m}\left(1 / \sigma_{-}\right)$is the sum of the first $m$ Fourier coefficients of $1 / \sigma_{-}$. This means we can evaluate $z^{n}\left(\psi^{m} / \sigma_{-}\right)_{[0, n]}(1)$ and then sum our evaluations. Now

$$
\begin{aligned}
\left(z^{n} \frac{\psi^{m}}{\sigma_{-}}\right)_{[0, n]}(1) & =\left(\frac{\psi^{m}}{\sigma_{-}}\right)_{[-n, 0]}(1)=\sum_{l=0}^{n} \sum_{k=1}^{\infty} \psi_{k}^{m} \xi_{k+l} \\
& =\sum_{k=1}^{\infty} \psi_{k}^{m} \sum_{l=0}^{n} \xi_{k+l}=\sum_{k=1}^{\infty} \psi_{k}^{m}\left[s_{k+n}\left(\frac{1}{\sigma_{-}}\right)-s_{k}\left(\frac{1}{\sigma_{-}}\right)\right] .
\end{aligned}
$$

A computation in $[16, \S \mathrm{VII}]$ shows that

$$
s_{N}\left(1 / \sigma_{-}\right)=\Gamma(1+\alpha-\beta)^{-1} N^{\alpha-\beta}+O\left(N^{-1 / 2-\delta}\right) .
$$

Thus

$$
\begin{aligned}
\left(z^{n} \frac{\psi}{\sigma_{-}}\right)_{[0, n]}(1)= & -\Gamma(1+\alpha-\beta)^{-1} \sum_{m=0}^{n} s_{m}\left(\frac{1}{\sigma_{-}}\right) \sum_{k=1}^{\infty} \psi_{k}^{m} \\
& \times\left[(k+n)^{\alpha-\beta}-k^{\alpha-\beta}+O\left(k^{-1 / 2-\delta}\right)\right] .
\end{aligned}
$$


The inner sum equals $\int_{0}^{\infty} \psi_{\{x\}}^{m}\left[(x+n)^{\alpha-\beta}-x^{\alpha-\beta}+O(1)\right]$, where, from Lemma 2.5,

$$
\begin{aligned}
\psi_{(x)}^{m}= & \frac{\Gamma(1-2 \beta) \sin \pi(\alpha+\beta)}{\pi}\left(I-K_{\alpha}\right)^{-1}(n x+m+1)^{-1+2 \beta}\left(\frac{x}{n}\right) \\
& +O(m+1)^{-1 / 2-\delta} .
\end{aligned}
$$

Using this expression for $\psi_{\{x\}}^{m}$ gives us for the inner sum

$$
\begin{gathered}
\frac{\Gamma(1-2 \beta) \sin \pi(\alpha+\beta)}{\pi} \int_{0}^{\infty}\left[\left(I-K_{\alpha}\right)^{-1}(n x+m+1)^{-1+2 \beta}\right]\left(\frac{x}{n}\right) \\
\times\left[(x+n)^{\alpha-\beta}-x^{\alpha-\beta}\right] d x \\
+O\left(n^{R \alpha+1 / 2}(m+1)^{-1 / 2-\delta}\right),
\end{gathered}
$$

and this $O$ refers to the $L_{2}$ norm. We can use Fubini now to interchange integration and we are left with

$$
\begin{aligned}
\left(z^{n} \frac{\psi}{\sigma_{-}}\right)_{[0, n]}(1) & =-\Gamma(1+\alpha-\beta)^{-1} \Gamma(1-2 \beta) \sin \pi(\alpha+\beta) \pi^{-1} \\
\times & \int_{0}^{\infty} \sum_{0}^{n} s_{m}\left(\frac{1}{\sigma_{-}}\right)\left(\left[\left(I-K_{\alpha}\right)^{-1}(n x+m+1)^{-1+2 \beta}\right]\left(\frac{x}{n}\right)\right) \\
& +O\left(n^{2 R \alpha+1-\delta}\right) .
\end{aligned}
$$

This uses the estimate for $S_{N}\left(1 / \sigma_{-}\right)$to estimate the last $O$ term. Using the above estimate again and the linearity of $\left(I-K_{\alpha}\right)^{-1}$ we can write the above integral as

$$
\begin{aligned}
& -\Gamma(1+\alpha-\beta)^{-2} \Gamma(1-2 \beta) \sin \pi(\alpha+\beta) \pi^{-1} n^{2 \alpha+1} \\
& \quad \times \int_{0}^{\infty}\left[\left(I-K_{\alpha}\right)^{-1} \sum_{0}^{n} \frac{1}{n}\left(\frac{m+1}{n}\right)^{\alpha-\beta}\left(x+\frac{m+1}{n}\right)^{-1+2 \beta}\right](x) \\
& \quad \times\left[(x+1)^{\alpha-\beta}-x^{\alpha-\beta}\right] d x \\
& \quad+O\left(n^{2 R \alpha+1-\delta}\right) .
\end{aligned}
$$

Finally we replace

$$
\sum_{0}^{n} \frac{1}{n}\left(\frac{m+1}{n}\right)^{\alpha-\beta}\left(x+\frac{m+1}{n}\right)^{-1+2 \beta} \text { by } \int_{0}^{1} \frac{t^{\alpha-\beta}}{(x+k)^{1-2 \beta}} d t .
$$

This can be justified using Minkowski's inequality for integrals. Then we can say that for $|R \alpha|<\frac{1}{2}$, 


$$
\begin{aligned}
\lim _{n \rightarrow \infty}( & \left.\frac{z^{n} \psi}{\sigma_{-}}\right)_{[0, n]}(1) / n^{2 \alpha+1}=-\Gamma(1+\alpha-\beta)^{-2} \Gamma(1-2 \beta) \sin \pi(\alpha+\beta) \pi^{-1} \\
& \times \int_{0}^{\infty}\left[\left(I-K_{\alpha}\right)^{-1} \int_{0}^{1} \frac{t^{\alpha-\beta}}{(x+t)^{1-2 \beta}} d t\right](x)\left[(x+1)^{\alpha-\beta}-x^{\alpha-\beta}\right] d x .
\end{aligned}
$$

To evaluate this integral we will return to the formulas for the inversion of $I-K_{\alpha}$, and for convenience we will call the integral $A$, and $\int_{0}^{1} t^{\alpha-\beta} /(x+$ $t)^{1-2 \beta} d t$ will be denoted by $g(x)$. Now recall that if $\left(I-K_{\alpha}\right) f=g$, then by (2.5), $\int_{0}^{\infty}(x+1)^{s-1} f(x) d x$ was $F_{-}(s)$, where

$$
\begin{aligned}
& F_{-}(s)=\frac{\Gamma(1-s-\alpha-\beta) \Gamma(1-s+\alpha-\beta)}{\Gamma(1-s) \Gamma(1-2 \beta-s)} G_{-}(s), \\
& G_{-}(s)=\frac{1}{2 \pi i} \int_{1 / 2-i \infty}^{1 / 2+i \infty} \frac{\Gamma(t-\alpha+\beta) \Gamma(t+\alpha+\beta)}{\Gamma(t) \Gamma(t+2 \beta)} \cdot \frac{G(t)}{t-s} d t, \quad R s<\frac{1}{2},
\end{aligned}
$$

and

$$
G(s)=\int_{0}^{\infty}(x+1)^{s-1} g(x) d x .
$$

One can then write $A$ using the binomial theorem as

$$
\begin{aligned}
\sum_{1}^{\infty} F_{-} & (\alpha-\beta-k+1) \frac{\Gamma(k-\alpha+\beta)}{\Gamma(k+1) \Gamma(\beta-\alpha)} \\
= & \sum_{1}^{\infty} \frac{\Gamma(k-2 \alpha) \Gamma(k) \Gamma(k-\alpha+\beta) G_{-}(\alpha-\beta-k+1)}{\Gamma(\beta-\alpha+k) \Gamma(k-\alpha-\beta) \Gamma(k+1) \Gamma(\beta-\alpha)} \\
= & \sum_{1}^{\infty} \frac{\Gamma(k-2 \alpha) G_{-}(\alpha-\beta-k+1)}{\Gamma(k-\alpha-\beta) k \Gamma(\beta-\alpha)}
\end{aligned}
$$

where $G_{-}$corresponds to this $g(x)$. The function $G(s)$ can be written in terms of a hypergeometric function and $G_{-}(s)$ can be computed using residues and the Beta function (see $[2,2.8,(46)])$. We leave the details to the reader. This allows us to write the integral $A$ as

$$
\begin{aligned}
& \frac{\Gamma(1+\alpha-\beta) \Gamma(1-\alpha-\beta) \Gamma(\alpha+\beta)}{\Gamma(\beta-\alpha) \Gamma(1-2 \beta)} \\
& \quad \times\left\{\sum_{1}^{\infty} \frac{-\Gamma(1+\alpha-\beta) \Gamma(k-1-2 \alpha)}{\Gamma(1+2 \alpha) k \Gamma(k-\alpha-\beta)}+\frac{\Gamma(k-1-2 \alpha)}{\Gamma(k+1)}\right\} .
\end{aligned}
$$

Now

$$
\sum_{1}^{\infty} \frac{\Gamma(k-1-2 \alpha)}{\Gamma(k+1)}=\frac{\Gamma(-2 \alpha)}{1+2 \alpha}
$$

and 


$$
\begin{aligned}
& \sum_{1}^{\infty} \frac{\Gamma(k-1-2 \alpha)}{k \Gamma(k-\alpha-\beta)} \\
& \quad=\frac{\Gamma(-2 \alpha)(-\alpha-\beta)}{\Gamma(1-\alpha-\beta)(1+2 \alpha)}\{\tilde{\psi}(\alpha-\beta+1)-\tilde{\psi}(-\alpha-\beta)\},
\end{aligned}
$$

where $\bar{\psi}$ is the logarithmic derivative of the gamma function. (See $[2,2.8,(46)]$ and $[2,17.4,(30)]$.) So

$$
\begin{aligned}
A= & \frac{\Gamma(1+\alpha-\beta) \Gamma(1-\alpha-\beta) \Gamma(\alpha+\beta) \Gamma(-2 \alpha)}{\Gamma(\beta-\alpha) \Gamma(1-2 \beta)(1+2 \alpha)} \\
& \times\left\{\frac{-\Gamma(1+\alpha-\beta)}{\Gamma(1+2 \alpha)} \frac{(-\alpha-\beta)}{\Gamma(1-\alpha-\beta)}[\tilde{\psi}(\alpha-\beta+1)-\tilde{\psi}(-\alpha-\beta)]+1\right\} .
\end{aligned}
$$

\section{Hence}

$$
\begin{aligned}
& \left(\frac{z^{n} \psi}{\sigma_{-}}\right)_{[0, n]}(1) / n^{2 \alpha+1}=\frac{\Gamma(-2 \alpha)}{\Gamma(\beta-\alpha)(1+2 \alpha)} \\
& \quad \times\left\{\frac{-(\alpha+\beta)}{\Gamma(1-\alpha-\beta) \Gamma(1+2 \alpha)}\right. \\
& \left.\quad \times[\tilde{\psi}(\alpha-\beta+1)-\tilde{\psi}(-\alpha-\beta)]-\frac{1}{\Gamma(1+\alpha-\beta)}\right\}+O\left(n^{-\delta}\right) \\
& =\frac{\Gamma(-2 \alpha)}{\Gamma(2+2 \alpha) \Gamma(\beta-\alpha) \Gamma(-\beta-\alpha)}[\tilde{\psi}(\alpha-\beta+1)-\tilde{\psi}(-\alpha-\beta)] \\
& \quad-\frac{\Gamma(-2 \alpha)}{(1+2 \alpha)} \frac{\sin \pi(\alpha-\beta)}{\pi}+O\left(n^{-\delta}\right) .
\end{aligned}
$$

Looking at (4.3) we still must estimate $\left(\phi / \sigma_{+}\right)_{[0, n]}(1)$ and $\left(q / \sigma_{-}\right)_{[0, n]}(1)$. But if then

$$
p \sigma=1+z+\cdots+z^{n}+\phi / \sigma_{+}+z^{n} \psi / \sigma_{-},
$$

$$
z^{n} p\left(\frac{1}{z}\right) \sigma\left(\frac{1}{z}\right)=1+z+\cdots+z^{n}+\frac{z^{n} \phi\left(z^{-1}\right)}{\sigma_{+}\left(z^{-1}\right)}+\frac{\psi\left(z^{-1}\right)}{\sigma_{-}\left(z^{-1}\right)} .
$$

Since $z^{n} p\left(z^{-1}\right)$ is a polynomial of degree $n, \phi\left(z^{-1}\right)$ corresponds to $\psi$ for $\sigma\left(z^{-1}\right)$. Replacing $z$ by $\left(z^{-1}\right)$ for our $\sigma$ only changes $\beta$ into $-\beta$. Thus we have

$$
\begin{aligned}
\left(\frac{\phi}{\sigma_{+}}\right)_{[0, n]} & (1) / n^{2 \alpha+1} \\
= & \frac{\Gamma(-2 \alpha)}{\Gamma(2+2 \alpha) \Gamma(\beta-\alpha) \Gamma(-\beta-\alpha)}[\tilde{\psi}(\alpha+\beta+1)-\tilde{\psi}(-\alpha+\beta)] \\
& -\frac{\Gamma(-2 \alpha)}{(1+2 \alpha)} \frac{\sin \pi(\alpha+\beta)}{\pi}+O\left(n^{-\delta}\right) .
\end{aligned}
$$


The computation of $(q / \sigma)_{[0, n]}(1)$ is straightforward. It comes from the observation that the Fourier coefficients of $\sigma^{-1}$ can be written as

$$
-\frac{\sin \pi(\alpha+\beta) \Gamma(1-2 \alpha)}{\pi} k^{-1+2 \alpha}+O\left(k^{-1+2 \alpha-\delta}\right) \text { for } k>0
$$

and

$$
-\frac{\sin \pi(\alpha-\beta) \Gamma(1-2 \alpha)}{\pi} k^{-1+2 \alpha}+O\left(k^{-1+2 \alpha-\delta}\right) \text { for } k<0 .
$$

Notice

$$
\left(\frac{q}{\sigma}\right)_{[0, n]}(1)=\sum_{-n}^{n}(n+1-|k|)\left(\frac{1}{\sigma}\right)_{k}
$$

So we have

$$
\begin{aligned}
\sum_{-n}^{n}(n+ & 1-|k|)\left(\frac{1}{\sigma}\right)_{k} \\
= & {\left[-\frac{\sin \pi(\alpha+\beta) \Gamma(1-2 \alpha)}{\pi(2 \alpha)(2 \alpha+1)}-\frac{\sin \pi(\alpha-\beta) \Gamma(1-2 \alpha)}{\pi(2 \alpha)(2 \alpha+1)}\right] n^{2 \alpha+1} } \\
& +O\left(n^{2 \alpha+1-\delta}\right) .
\end{aligned}
$$

We have now evaluated all three of the terms in (4.3). Putting them together we have

$$
\begin{aligned}
& p_{[0, n]}(1) / n^{2 \alpha+1}= \frac{\Gamma(-2 \alpha)}{\Gamma(2+2 \alpha) \Gamma(\beta-\alpha) \Gamma(-\beta-\alpha)} \\
& \times[\tilde{\psi}(\alpha-\beta+1)-\tilde{\psi}(-\alpha-\beta)]-\frac{\Gamma(-2 \alpha) \sin \pi(\alpha-\beta)}{(1+2 \alpha) \pi} \\
& \quad+\frac{\Gamma(-2 \alpha)}{\Gamma(2+2 \alpha) \Gamma(\beta-\alpha) \Gamma(-\beta-\alpha)}[\tilde{\psi}(\alpha+\beta+1)-\tilde{\psi}(-\alpha+\beta)] \\
& \quad-\frac{\Gamma(-2 \alpha) \sin \pi(\alpha+\beta)}{(1+2 \alpha) \pi}-\frac{\sin \pi(\alpha+\beta) \Gamma(1-2 \alpha)}{\pi(2 \alpha)(2 \alpha+1)} \\
& \quad-\frac{\sin (\alpha-\beta) \Gamma(1-2 \alpha)}{\pi(2 \alpha)(2 \alpha+1)}+O\left(n^{-\delta}\right) \\
&= \frac{\Gamma(1+\alpha-\beta) \Gamma(1+\alpha+\beta) \sin \pi(\alpha+\beta) \sin \pi(\alpha-\beta)}{\Gamma(1+2 \alpha) \Gamma(2+2 \alpha) \pi(-\sin 2 \pi \alpha)} \\
& \times[\tilde{\psi}(\alpha-\beta+1)-\tilde{\psi}(-\alpha-\beta)+\tilde{\psi}(\alpha+\beta+1)-\tilde{\psi}(-\alpha+\beta)] \\
&+O\left(n^{-\delta}\right) .
\end{aligned}
$$


Now $\psi(1-z)-\psi(z)=\pi \cot (\pi z)[$ see $2,1.7 .1,(11)]$. So the term in brackets is

$$
\begin{aligned}
& -\pi\left[\frac{\cos \pi(\alpha+\beta)}{\sin \pi(\alpha+\beta)}+\frac{\cos \pi(\alpha-\beta)}{\sin \pi(\alpha-\beta)}\right] \\
& \quad=-\pi\left[\frac{\cos \pi(\alpha+\beta) \sin \pi(\alpha-\beta)+\cos \pi(\alpha-\beta) \sin \pi(\alpha+\beta)}{\sin \pi(\alpha+\beta) \sin \pi(\alpha-\beta)}\right] \\
& \quad=\pi \frac{-\sin 2 \pi \alpha}{\sin \pi(\alpha+\beta) \sin \pi(\alpha-\beta)} .
\end{aligned}
$$

Substituting this in the bracket we have

$$
\begin{aligned}
p_{[0, n]}(1) / n^{2 \alpha+1} & =\left(T_{n}^{-1}[\sigma] q\right)(1) / n^{2 \alpha+1} \\
& =\frac{\Gamma(1+\alpha-\beta) \Gamma(1+\alpha+\beta)}{\Gamma(1+2 \alpha) \Gamma(2+2 \alpha)}+O\left(n^{-\delta}\right) .
\end{aligned}
$$

The substitution of this in (4.2) proves the proposition.

From (2.1) and Proposition (4.1) we know that for $|R \alpha|<\frac{1}{2}$ and $-\frac{1}{2}<R \alpha<\frac{3}{2}$ the limit of $D_{n}[\alpha] n^{-\alpha^{2}+\beta^{2}}$ exists and satisfies a difference equation.

5. Final result. In this section we will first show $\lim _{n \rightarrow \infty} S_{n}[\sigma]$ exists for $R \alpha_{r}>-\frac{1}{2}$. We begin by proving Proposition 5.1.

Proposition 5.1. $S_{n}[\sigma]=O(1)$ uniformly in any compact subset of $R \alpha_{r}>-\frac{1}{2}$.

If $D_{n}[\sigma]$ is the Toeplitz determinant for $\sigma$ which $\left|R \alpha_{r}\right|<\frac{1}{2}$, we want to replace $\alpha_{r}$ by $\alpha_{r}+k_{r}$ where $k_{r}=0,1, \ldots$ For simplicity's sake let us suppose each $k_{r}$ is 0 or 1 . Using (4.1) we have the identity

$$
\begin{aligned}
D_{n-p} & {\left[\sigma \prod_{r=1}^{P}\left(1-\frac{z}{z_{r}}\right)\left(1-\frac{z_{r}}{z}\right)\right] } \\
& =\prod_{1<r<s<p}\left|z_{r}-z_{s}\right|^{-2} D_{n}[\sigma]\left|\left[T_{n}[\sigma]^{-1}\left(\sum_{k=0}^{n} z_{s}^{-k} z^{k}\right)\right]\left(z_{r}\right)\right|_{r, s=1, \ldots, p} .
\end{aligned}
$$

Thus we need to estimate $p=T_{n}^{-1}[\sigma] q$ at $z=z_{r}$ where

$$
q=\sum_{k=0}^{n} z_{s}^{-k} z^{k}
$$

As in the previous section one has 


$$
p=p_{[0, n]}=\left(\frac{q}{\sigma}\right)_{[0, n]}+\left(\frac{\phi}{\sigma_{+}}\right)_{[0, n]}+\left(z^{n} \frac{\psi}{\sigma_{-}}\right)_{[0, n]} .
$$

Using the techniques of the last section where we found the difference equation and referring to details in [16, VIII], one can easily show that both of the terms $\left(z^{n} \psi / \sigma_{-}\right)_{[0, n]}\left(z_{r}\right)$ and $\left(\phi / \sigma_{+}\right)_{[0, n]}\left(z_{r}\right)$ are $O\left(n^{R\left(\alpha_{r}+\alpha_{s}\right)+1}\right)$. We must also estimate $(q / \sigma)_{[0, n]}\left(z_{r}\right)$ for $q=\sum_{k=0}^{n} z_{s}^{-k_{z} z^{k}}$. Referring again to [16, VIII] one has $\left(z=e^{i \theta}, z_{r}=e^{i \theta_{r}}, z_{s}=e^{i \theta_{s}}\right)$

$$
\begin{aligned}
\left(\frac{q}{\sigma}\right)_{[0, n]}\left(z_{r}\right) & =\frac{1}{2 \pi} \int_{-\pi}^{\pi} \sigma(z) \frac{\left(z / z_{s}\right)^{n+1}-1}{z / z_{s}-1} \frac{\left(z_{r} / z\right)^{n+1}-1}{z_{r} / z-1} d \theta \\
& =O\left(n^{R\left(\alpha_{r}+\alpha_{s}\right)+1}\right) \text { for } R \alpha_{r}, R \alpha_{s} \neq 0 .
\end{aligned}
$$

The constant implied in this $O$ is $O\left(\left|R \alpha_{r}\right|^{-1}+\left|R \alpha_{s}\right|^{-1}\right)$.

From these estimates we have for $R \alpha_{r}, R \alpha_{s} \neq 0$,

$$
T_{n}[\sigma]^{-1}\left(\sum z_{s}^{-k} z^{k}\right)\left(z_{r}\right)=O\left(n^{R\left(\alpha_{r}+\alpha_{s}\right)+1}\right),
$$

where again the constant implied in the $O$ is $O\left(\left|R \alpha_{r}\right|^{-1}+\left|R \alpha_{s}\right|^{-1}\right)$. And so

$$
\left|T_{n}[\sigma]^{-1}\left(\sum z_{s}^{-k} z^{k}\right)\left(z_{r}\right)\right|_{r, s=1, \ldots, p}=O\left(n^{\Sigma\left\{2 R \alpha_{r}+1\right.}\right) .
$$

The constant implied in this last $O$ is $\Pi_{1}^{P}\left(R \alpha_{r}\right)^{-2}$. This can be seen by factoring $\left(R \alpha_{r}\right)^{-1}$ and $\left(R \alpha_{s}\right)^{-1}$ out of the $r$ th row and sth column, respectively.

Hence from (2.14) it follows that for $\left|R \alpha_{r}\right|<\frac{1}{2}$ and $\left|R \alpha_{r}\right| \neq 0, D_{n}\left[\sigma \Pi_{1}^{p}(1-\right.$ $\left.\left.z / z_{r}\right)\left(1-z_{r} / z\right)\right]$ is at most a constant times

$$
n^{\Sigma\left(\alpha_{r}^{2}-\beta_{r}^{2}\right)+\Sigma p\left(2 \alpha_{r}+1\right)}=n^{\Sigma_{i}^{R}\left(\alpha_{r}+k_{r}\right)^{2}-\beta_{r}^{2}} .
$$

The extension of this result to arbitrary nonnegative $k_{r}$ can be done with slightly more complicated calculations.

We still must extend this result to the lines $R \alpha_{r}=a$ nonnegative integer and $R \alpha_{r}=$ a nonnegative integer $+\frac{1}{2}$. These lines are taken care of by the following lemma found in [16, VIII].

Suppose $h$ is analytic on $|z|<\frac{1}{2}$ and satisfies there $|h(z)|<|R z|^{-c}$, where $c$ is a positive constant. Then for each subdisc $|z|<p\left(p<\frac{1}{2}\right)$ we have $|h(z)|<A$, where $A$ is a constant depending only on $c$ and $p$.

By using the estimates obtained in this section and the fact that $S_{n}[\sigma]$ is uniformly bounded for $\left|R \alpha_{r}\right|<\frac{1}{2}$ and $\left|\alpha_{r}\right|$ bounded, we have $S_{n}[\sigma]=$ $O\left(I d_{r}^{-2}\right)$, where $d_{r}$ is the distance from $\alpha_{r}$ to the nearest exceptional line. The left side of this equation is analytic in the $\alpha_{r}$. Hence, by repeated applications of the lemma (with $c=2$ and the center of the disc on $R \alpha_{r}=\frac{1}{2}, 1$ ) we obtain $S_{n}[\sigma]=O(1)$ uniformly in any compact subset of $R \alpha_{r}>-\frac{1}{2}$ and the proposition is proved. 
Combining Proposition 5.1 with (2.1) yields the existence of the limit $S_{n}[\sigma]$, for $R \alpha_{r}>-\frac{1}{2}$, which is an analytic function of the $\alpha_{r}$ 's. In particular we know that the limit of $D_{n}[\alpha] n^{-\left(\alpha^{2}-\beta^{2}\right)}$ exists for $R \alpha>-\frac{1}{2}$. Call this limit $E_{\alpha, \beta}$. Now we need to recall the results of $\S 4$. There it was shown that for $\alpha_{r}$ real and $\left|\alpha_{r}\right|<\frac{1}{2}$,

$$
D_{n}[\sigma]=\left(D_{n}[\sigma] / D_{n-1}[\sigma]\right)^{n+1} E(n),
$$

where $E(n)$ is given by (3.2) and equals

$$
\begin{aligned}
E[\tau] & \prod_{r=1}^{R} \tau_{+}\left(\theta_{r}\right)^{-\alpha_{r}+\beta_{r} \tau_{-}}\left(\theta_{r}\right)^{-\alpha_{r}-\beta_{r}} \\
& \times \prod_{r \neq s}\left(1-e^{i\left(\theta_{s}-\theta_{r}\right)}\right)^{-\left(\alpha_{r}+\beta_{r}\right)\left(\alpha_{s}-\beta_{s}\right)} \exp \left\{\sum_{r=1}^{R} F\left(n, \alpha_{r}, \beta_{r}\right)\right\} .
\end{aligned}
$$

Recall also that from (2.1) for $\left|R \alpha_{r}\right|<\frac{1}{2}$,

$$
\left(\frac{D_{n}[\sigma]}{D_{n-1}[\sigma]}\right)^{n+1}=\exp \left\{\sum \alpha_{r}^{2}-\beta_{r}^{2}+O\left(n^{-\delta}\right)\right\}
$$

Therefore for $D_{n}[\alpha]$ with $\alpha$ real and $|\alpha|<\frac{1}{2}$, we have

$$
\lim _{n \rightarrow \infty} D_{n}[\alpha] n^{-\alpha^{2}-\beta^{2}}=\lim _{n \rightarrow \infty} \exp \left\{\alpha^{2}-\beta^{2}+F(n, \alpha, \beta)\right\} n^{-\alpha^{2}+\beta^{2}}=E_{\alpha, \beta} \text {. }
$$

Thus $e^{F\left(n, \alpha_{r}, \beta_{r}\right)}$ is determined asymptotically in terms of $E\left(\alpha_{r}, \beta_{r}\right)$. Substitution into (5.1) therefore gives for $\alpha_{r}$ real and $\left|\alpha_{r}\right|<\frac{1}{2}$,

$$
\begin{aligned}
\lim _{n \rightarrow \infty} S_{n}[\sigma]= & E[\tau] \prod_{r=1}^{R} \tau_{+}\left(\theta_{r}\right)^{-\alpha_{r}+\beta_{r}} \tau_{-}\left(\theta_{r}\right)^{-\alpha_{r}-\beta_{r}} \\
& \times \prod_{r \neq s}\left(1-e^{i\left(\theta_{s}-\theta_{r}\right)}\right)^{-\left(\alpha_{r}+\beta_{r}\right)\left(\alpha_{s}-\beta_{s}\right)} \prod_{r=1}^{R} E_{\alpha_{r}, \beta_{r}}
\end{aligned}
$$

Since the right-hand side of the equation is an analytic function of the $\alpha_{r}$ 's for $R \alpha_{r}>-\frac{1}{2}$, and since the limit of $S_{n}[\sigma]$ exists and is analytic for $R \alpha_{r}>-\frac{1}{2}$, the above holds for all $\alpha_{r}$ satisfying $R \alpha_{r}>-\frac{1}{2}$.

From Proposition 4.1 we have

$$
\frac{E_{\alpha+1, \beta}}{E_{\alpha, \beta}}=\frac{\Gamma(1+\alpha-\beta) \Gamma(1+\alpha+\beta)}{\Gamma(1+2 \alpha) \Gamma(2+2 \alpha)} .
$$

The rest of this section will be devoted to proving that

$$
E_{\alpha, \beta}=G(1+\alpha+\beta) G(1+\alpha-\beta) / G(1+2 \alpha)
$$

where $G$ is the Barnes $G$-function. The proof of this is divided into several lemmas. 
LEMMA 5.2. Let

$$
Q(\alpha)=E_{\alpha, \beta} G(1+2 \alpha) / G(1+\alpha-\beta) G(1+\alpha+\beta) .
$$

Then $Q(\alpha)$ is an entire periodic function of $\alpha$ with period one and $Q(\alpha)$ has no zeros.

PROOF. $E_{\alpha, \beta}$ is analytic for $R \alpha>-\frac{1}{2}$ and the zeros of $G(1+\alpha \pm \beta)$ occur at $\alpha=\mp \beta-k, k=-1,-2, \ldots$. Hence $Q(\alpha)$ is analytic for $R \alpha>-\frac{1}{2}$. From $\S 4$ and [1] we know $E_{\alpha, \beta}$ and $G(1+2 \alpha) / G(1+\alpha-\beta) G(1+\alpha+\beta)$ satisfy the same difference equation for $R \alpha>-\frac{1}{2}$. Hence their quotient must be a periodic function with period one, and since $Q(\alpha)$ is analytic for $R \alpha>-\frac{1}{2}$, it is actually entire. Finally $G(1+2 \alpha)$ has no zeros for $R \alpha>-\frac{1}{2}$, and since $E_{\alpha, \beta}$ is given by an exponential (using the preliminary form in $\S 3$ ) for $R \alpha>-\frac{1}{2}$, we can conclude that $Q(\alpha)$ has no zeros.

Our next step is to show that $\log Q(\alpha)$ is an entire function of the exponential type. We need the following technical lemma in what follows.

LEMMA 7.3. Suppose $\sigma(\theta)=\left(1-e^{i \theta}\right)^{\alpha+\beta}\left(1-e^{-i \alpha}\right)^{\alpha-\beta}$. Then for $|R \alpha|<\frac{1}{2}$ the $O$ in (2.1) in $O\left(e^{3 \pi|\alpha|}\right)$, and this last $O$ is independent of $\alpha$. The $\delta$ in (2.1) in this special case is 1 . In addition we can say that (2.1) holds for $n>N_{\alpha}$, where $N_{\alpha}$ can be chosen to be $O\left(e^{(3 \pi+\varepsilon)|\alpha|}\right)(\varepsilon>0)$.

In order to do this, we retrace our estimates of $\S 2$. Recall that the $O$ in (2.1) was given by integrating from $O$ to $\infty$ the product of (here $R=1$ )

$$
\begin{aligned}
\psi_{\{x\}}= & \frac{-\Gamma(1-2 \beta) \sin \pi(\alpha+\beta)}{\pi}\left(I-K_{\alpha}\right)^{-1}(n x+n)^{-1+2 \beta}\left(\frac{x}{n}\right) \\
& +O\left(n^{-1 / 2-\delta}\right)
\end{aligned}
$$

and

$$
\xi_{\{x\}+n}=\frac{\Gamma(1+2 \beta) \sin \pi(\alpha-\beta)}{\pi(x+n)^{1+2 \beta}}+O\left((x+n)^{-1-\delta}\right) .
$$

The $O$ 's above referred to the $L_{2}$ norm. Thus we must investigate the $O$ 's in these two terms. Now in the case we have now

$$
\sigma(\theta)=\left(1-e^{i \theta}\right)^{\alpha+\beta}\left(1-e^{-i \theta}\right)^{\alpha-\beta},
$$

and we can make the formulas for $\psi_{\{x\}}$ and $\zeta_{\{x\}+n}$ more precise. One can easily show that for $k>0$,

$$
\frac{1}{2 \pi} \int_{-\pi}^{\pi} \frac{\sigma_{+}}{\sigma_{-}} e^{i k \theta} d \theta=\frac{\sin \pi(\alpha-\beta)}{\pi} \frac{\Gamma(1+2 \beta) \Gamma(k+\alpha-\beta+1)}{\Gamma(k+\alpha+\beta+2)}
$$

and 


$$
\frac{1}{2 \pi} \int_{-\pi}^{\pi} \frac{\sigma_{-}}{\sigma_{+}} e^{-i k \theta} d \theta=\frac{\sin \pi(\alpha+\beta)}{\pi} \frac{\Gamma(1-2 \beta) \Gamma(k+\alpha+\beta+1)}{\Gamma(k+\alpha-\beta+2)} .
$$

Now an expansion of the form $\Gamma(z+A) / \Gamma(z+B)$ has an asymptotic expansion $[2,1.18,(4)]$

$$
z^{A-B}\left[1+\frac{1}{2} z^{-1}(A-B)(A-B+1)+O\left(z^{-2}\right)\right], \quad|z| \rightarrow \infty .
$$

Thus one can then say as $k \rightarrow \infty$,

$$
\begin{aligned}
\frac{1}{2 \pi} \int_{-\pi}^{\pi} \frac{\sigma_{+}}{\sigma_{-}} e^{i k \theta} d \theta= & \frac{\sin \pi(\alpha-\beta) \Gamma(1+2 \beta)}{\pi} k^{-1-2 \beta} \\
& +\left(O|\alpha| k^{-2}\right)
\end{aligned}
$$

and

$$
\begin{aligned}
\frac{1}{2 \pi} \int_{-\pi}^{\pi} \frac{\sigma_{-}}{\sigma_{+}} e^{-i k \theta} d \theta= & \frac{\sin \pi(\alpha+\beta) \Gamma(1+2 \beta)}{\pi} k^{-1+2 \beta} \\
& +\left(O|\alpha| k^{-2}\right)
\end{aligned}
$$

and the $O$ 's in these expressions are independent of $\alpha$. Thus retracing our estimates for $\zeta_{\{x\}+n}$ and using the fact that $|\sin \pi(\alpha+\beta)|<A e^{\pi|\alpha|}$, where $A$ is some constant independent of $\alpha$, we have that

$$
\zeta_{\{x\}+n}=\frac{\Gamma(1+2 \beta) \sin \pi(\alpha-\beta)}{\pi(x+n)^{1+2 \beta}}+O\left(|\alpha| e^{\pi|\alpha|}(x+n)^{-1-\delta}\right),
$$

and this $O$ depends only on $\beta$.

We now turn to the estimates for $\psi_{\{x\}}$. The term $\psi_{\{x\}}$ was given by $\left[(I-K)^{-1}+O\left(n^{-\delta}\right)\right] g(x)$, where

$$
g(x)=\frac{\Gamma(1-2 \beta) \sin \pi(\alpha+\beta)}{\pi(x+n)^{1-2 \beta}}+O\left(n^{-1 / 2-\delta}\right) .
$$

Again these $O$ 's refer to the $L_{2}$ norm. The $O$ in the expression for $g(x)$ is $O\left(e^{\pi|\alpha|} n^{-1 / 2-\delta}\right)$. This can be seen by computations that are similar to the previous ones done for $\zeta_{\{x\}+n}$. The $O$ in the expression for the operator came from the norm of $\left(I-K_{\alpha}\right)^{-1}\left(I-\left(I-K_{\alpha}\right)^{-1} K_{e}\right)^{-1}$. From (5.2)-(5.3) one can see that the norm of $K_{e}$ contributes $O\left(\alpha^{2} e^{2 \pi|\alpha|}\right)$. We now concentrate on the norm of $\left(I-K_{\alpha}\right)^{-1}$. From the inversion formulas for $\left(I-K_{\alpha}\right)^{-1}$ one has that

$$
\left\|\left(I-K_{\alpha}\right)^{-1}\right\| \leqslant\left\|\frac{\Gamma(t-\alpha+\beta) \Gamma(t+\alpha+\beta)}{\Gamma(t) \Gamma(t+2 \beta)}\right\|_{\infty}^{2}
$$

where the $\infty$ norm is taken on the line $R t=\frac{1}{2}$. Hence we must estimate $\|\Gamma(t-\alpha+\beta) \Gamma(t+\alpha+\beta) / \Gamma(t) \Gamma(t+2 \beta)\|_{\infty}$ for $\alpha$ sufficiently large. Our 
estimates are concerned with the usual infinity norm and they are straightforward if one uses $[2,1.18,(6)]$

$$
\lim _{|y| \rightarrow \infty}|\Gamma(x+i y)| e^{\pi|y| / 2}|y|^{1-x / 2}=\left(2 \pi^{1 / 2}\right) .
$$

One can then easily see that

$$
\left\|\left(I-K_{\alpha}\right)^{-1}\right\|=O\left(e^{-\pi|\alpha|}\right) .
$$

These estimates however are not valid if $R \alpha=\frac{1}{2}$ or $R \alpha=-\frac{1}{2}$. But by moving the line of integration to the left in (2.5) as we did for (2.14) we can see that these estimates are uniform for $|R \alpha|<\frac{1}{2}$ and $|\alpha|$ sufficiently large. Hence we can conclude that uniformly in $|R \alpha|<\frac{1}{2}$ for $\alpha$ sufficiently large

$$
\begin{aligned}
\psi_{(x)}= & -\frac{\Gamma(1-2 \beta) \sin \pi(\alpha+\beta)}{\pi}\left(I-K_{\alpha}\right)^{-1}-(n x+n)^{-1+2 \beta}\left(\frac{x}{n}\right) \\
& +O\left(e^{2 \pi|\alpha|} n^{-1 / 2-\delta}\right) .
\end{aligned}
$$

Putting all of this together we have shown that the $O$ in $(2.1)$ is $O\left(e^{3 \pi|a|}\right)$. The statements about the $\delta$ and $N$ follow by retracing our estimates for $\delta$ in the above proof and using the just obtained estimate for the $O$ in (2.1). that

From this result we will now get estimates for $E_{\alpha, \beta}$ with $|R \alpha|<\frac{1}{2}$. Recall

$$
E_{\alpha, \beta}=\lim _{n \rightarrow \infty} D_{n}[\alpha] n^{-\alpha^{2}+\beta^{2}}, \quad R \alpha>-\frac{1}{2} .
$$

We can now estimate $\lim _{n \rightarrow \infty} D_{n}[\alpha] n^{-\alpha^{2}+\beta^{2}}$ using (2.1). Suppose now that (2.1) holds for $n>N_{\alpha}$. Then from (2.1) we have

$$
\frac{D_{N}[\alpha]}{D_{N_{\alpha}}[\alpha]}=\prod_{N_{\alpha}}^{N}\left(1-n^{-1}\left(\alpha^{2}-\beta^{2}\right)+O\left(n^{-1-\delta}\right)\right) \text {. }
$$

So

$$
\begin{aligned}
\log D_{N}[\alpha]-\log D_{N_{\alpha}}[\alpha] & =\sum_{N_{\alpha}}^{N} \log \left(1-n^{-1}\left(\alpha^{2}-\beta^{2}\right)+O\left(n^{-1-\delta}\right)\right) \\
& =\sum_{N_{\alpha}}^{N} n^{-1}\left(\alpha^{2}-\beta^{2}\right)+O\left(n^{-1-\delta}\right) \\
& =\left(\alpha^{2}-\beta^{2}\right)\left(\log N-\log N_{\alpha}\right)+O\left(N_{\alpha}^{-\delta}\right) .
\end{aligned}
$$

Hence

$$
D_{N}[\alpha] N^{-\alpha^{2}+\beta^{2}}=D_{N_{\alpha}}[\alpha] N_{\alpha}^{-\alpha^{2}+\beta^{2}} \exp \left\{O\left(N_{\alpha}^{-\delta}\right)\right\} .
$$

This last $O$ is still $O\left(e^{3 \pi \mid \alpha}\right)$. Now because of Lemma 5.3 we can choose $N_{\alpha}$ to be $O\left(e^{(3 \pi+\varepsilon)|\alpha|}\right)(\varepsilon>0)$ for all $|\alpha|$ sufficiently large. Hence one has 


$$
D_{N}[\sigma] N^{-\alpha^{2}+\beta^{2}}=O\left(D_{N_{\alpha}}[\alpha] e^{|p(\alpha)|}\right)
$$

where $p(\alpha)$ is again some polynomial.

This last formula implies that we must still estimate $D_{N_{\alpha}}[\alpha]$. This is done in the following

LEMMA 5.4.

$$
D_{N_{\alpha}}[\alpha]=O\left(\exp e^{(4 \pi+\varepsilon)|\alpha|}\right) .
$$

Proof. We use now the formula from [15],

$$
T_{n}[\sigma] T_{n}\left[\sigma_{-}^{-1}\right] T_{n}\left[\sigma_{+}^{-1}\right]=I_{n}-P_{n} H[\sigma] H\left[\tilde{\sigma}_{-}^{-1}\right] P_{n} T_{n}\left[\sigma_{+}^{-1}\right]
$$

Here we are using the notation

$$
T_{n}[\phi]=\left(\phi_{i-j}\right), \quad H[\phi]=\left(\phi_{i+j+1}\right), \quad i, j=0, \ldots, n,
$$

$\tilde{\phi}(z)=\phi\left(z^{-1}\right)$ and $I_{n}$ and $P_{n}$ are the identity and projection matrices on $C^{n}$, respectively. The determinant on the left-hand side of $(5.6)$ is $D_{n}[\alpha]$. The right-hand side of (5.6) has determinant

$$
\operatorname{Det}\left[I_{n}-P_{n} H[\sigma] H\left[\tilde{\sigma}_{-}^{-1}\right] P_{n} T_{n}\left[\sigma_{+}^{-1}\right]\right] \text {. }
$$

Using the inequality $[7, \mathrm{IV} \S 1,(1.2)]$

$$
|\operatorname{Det}[I-A]| \leqslant e^{|A|} \text {, }
$$

where $|\cdot|_{1}$ is the trace norm of the operator $A$, one has

$$
D_{n}[\sigma] \leqslant \exp \left|H_{n}[\sigma] H_{n}\left[\tilde{\sigma}_{-}^{-1}\right] T_{n}\left[\sigma_{+}^{-1}\right]\right|_{1} \text {, }
$$

where $H_{n}$ now means $H_{n}[\phi]=\left(\phi_{i+j+1}\right), i, j=0, \ldots, n$. The Schwarz inequality for operators gives

$$
D_{n}[\sigma] \leqslant \exp \left\|H_{n}[\sigma]\right\|_{2}\left\|H_{n}\left[\tilde{\sigma}_{-}^{-1}\right]\right\|_{2}\left\|T_{n}\left[\sigma_{+}^{-1}\right]\right\|_{\infty},
$$

where \|\|$_{\infty}$ is the usual operator norm, and \|\|$_{2}$ is the Hilbert Schmidt norm. For definitions of these norms and proofs of the inequalities, see [7, Chapters II and IV]. Thus in order to estimate $D_{n}[\alpha]$ we will estimate these three norms. We need again the estimates for the coefficients of $\sigma, \tilde{\sigma}_{-}^{-1}$ and $\sigma_{+}^{-1}$. These coefficients can again be found exactly in this case. We have for $k>0$,

$$
\begin{gathered}
\frac{1}{2 \pi} \int_{-\pi}^{\pi} \sigma e^{-i k \theta} d \theta=\frac{-\Gamma(1+2 \alpha) \sin \pi(\alpha+\beta)}{\pi} \frac{\Gamma(k-\alpha-\beta)}{\Gamma(k-\beta+\alpha+1)}, \\
\frac{1}{2 \pi} \int_{-\pi}^{\pi} \tilde{\sigma}_{-}^{-1} e^{-i k \theta} d \theta=\frac{\Gamma(\alpha-\beta+k)}{\Gamma(\alpha-\beta) \Gamma(k+1)} \\
\frac{1}{2 \pi} \int_{-\pi}^{\pi} \sigma_{+}^{-1} e^{i k \theta} d \theta=0 \\
\frac{1}{2 \pi} \int_{-\pi}^{\pi} \sigma_{+}^{-1} e^{-k \theta} d \theta=\frac{\Gamma(\alpha+\beta+k)}{\Gamma(\alpha+\beta) \Gamma(k+1)} .
\end{gathered}
$$


Using the asymptotic formula for $\Gamma(z+A) / \Gamma(z+B)$ we have for $k>0$,

$$
\begin{gathered}
\frac{1}{2 \pi} \int_{-\pi}^{\pi} \sigma e^{-i k \theta} d \theta=\frac{-\Gamma(1+2 \alpha) \sin \pi(\alpha+\beta)}{\pi}\left[|k-\beta|^{-1-2 \alpha}+O\left(k^{-2}\right)\right], \\
\frac{1}{2 \pi} \int_{-\pi}^{\pi} \tilde{\sigma}_{-}^{-1} e^{-i k \theta} d \theta=\frac{1}{\Gamma(\alpha-\beta)}\left[|k|^{-1+\alpha}+O\left(k^{-2}\right)\right] .
\end{gathered}
$$

From these estimates we can say

$\left\|H_{n}[\sigma]\right\|_{2}^{2}=O\left(\left|\frac{\Gamma(1+2 \alpha) \sin \pi(\alpha+\beta)}{\pi}\right|^{2} \sum_{1}^{n}\left[k|k-\beta|^{-2-4 \alpha}+O\left(k^{-2}\right)\right]\right]$.

Hence,

$$
\left\|H_{n}[\sigma]\right\|_{2}= \begin{cases}o\left(\left|\frac{\Gamma(1+2 \alpha) \sin \pi(\alpha+\beta)}{\pi} n^{-2 R \alpha}\right|\right), & R \alpha<0, \\ o\left(\left|\frac{\Gamma(1+2 \alpha) \sin \pi(\alpha+\beta)}{\pi} \log n\right|\right), & R \alpha=0, \\ O\left(\mid \frac{\Gamma(1+2 \alpha) \sin \pi(\alpha+\beta)}{\pi} \|\right), & R \alpha>0 .\end{cases}
$$

Similarly,

$$
\left\|H_{n}\left[\tilde{\sigma}_{-}^{-1}\right]\right\|_{2}= \begin{cases}O\left(|1 / \Gamma(\alpha-\beta)| n^{R \alpha}\right), & R \alpha>0 \\ O(|1 / \Gamma(\alpha-\beta)| \log n), & R \alpha=0 \\ O(|1 / \Gamma(\alpha-\beta)|), & R \alpha<0 .\end{cases}
$$

In order to estimate (5.6) we still need to estimate $\left\|T_{n}\left[\sigma_{+}^{-1}\right]\right\|_{\infty}$. For $R \alpha \leqslant$ $0, \sigma_{+}^{-1}$ is bounded, and since

$$
\left\|T_{n}\left[\sigma_{+}^{-1}\right]\right\|_{\infty} \leqslant\left\|\sigma_{+}^{-1}\right\|_{\infty}
$$

where this last infinity norm refers to the usual sup norm on $S^{1}$, we can say

$$
\left\|T_{n}\left[\sigma_{+}^{-1}\right]\right\|_{\infty}=O\left(e^{\pi|\alpha| / 2}\right) .
$$

For $R \alpha>0$ we replace $\sigma_{+}^{-1}$ in $T_{n}\left[\sigma_{+}^{-1}\right]$ by the function $\sum_{-n}^{n}\left(\sigma_{+}^{-1}\right)_{k} e^{i k \theta}$. Recall $\left(\sigma_{+}^{-1}\right)_{k}$ is the $k$ th Fourier coefficient of $\sigma_{+}^{-1}$. Clearly the norm of $\left\|T_{n}\left[\sigma_{+}^{-1}\right]\right\|_{\infty}$ is not changed by this replacement.

$$
\left\|T_{n}\left[\sigma_{+}^{-1}\right]\right\|_{\infty} \leqslant\left\|\sum_{-n}^{n}\left(\sigma_{+}^{-1}\right)_{k} e^{i k \theta}\right\|_{\infty},
$$

where the last infinity norm refers to the usual $\infty$-norm. Now 


$$
\begin{aligned}
\left(\sigma_{+}^{-1}\right)_{k} & =\Gamma(\alpha+\beta+k) / \Gamma(\alpha+\beta) \Gamma(k+1) \\
& =(1 / \Gamma(\alpha+\beta))\left[|k|^{\alpha+\beta-1}+O|k|^{-2}\right] .
\end{aligned}
$$

Hence,

$$
\left|\sum_{-n}^{n}\left(\sigma_{+}^{-1}\right)_{k}\right|=O\left(\frac{1}{\Gamma(\alpha+\beta)} n^{R \alpha}\right)
$$

and

$$
\left\|T_{n}\left[\sigma_{+}^{-1}\right]\right\|_{\infty}=O\left(\frac{1}{\Gamma(\alpha+\beta)} n^{R \alpha}\right) .
$$

Putting the estimates for $\left\|H_{n}[\sigma]\right\|_{2},\left\|H_{n}\left[\tilde{\sigma}_{-}^{-1}\right]\right\|_{2}$ and $\left\|T_{n}\left[\sigma_{+}^{-1}\right]\right\|_{\infty}$ together with (5.7) we have

$$
D_{N_{\alpha}}=\left\{\begin{array}{l}
\left.o\left[\exp \left[\frac{\sin \pi(\alpha+\beta)}{\pi} \frac{\Gamma(1+2 \alpha)}{\Gamma(\alpha+\beta) \Gamma(\alpha-\beta)} N_{\alpha}^{2 R \alpha}\right]\right]_{\text {for } R \alpha \geqslant 0,}\right] \\
O\left[\exp \left[\frac{\sin \pi(\alpha+\beta)}{\pi} \frac{\Gamma(1+2 \alpha)}{\Gamma(\alpha+\beta)} e^{\pi|\alpha| / 2} N_{\alpha}^{2|R \alpha|}\right]\right]_{\text {for } R \alpha<0 .}
\end{array}\right.
$$

Using the asymptotic expansion for $\Gamma(x+i y),|y| \rightarrow \infty$, and the fact that $|R \alpha|<\frac{1}{2}$, we have

$$
D_{N_{\alpha}}=O\left(\exp e^{\pi|\alpha|} N_{\alpha}\right)
$$

Hence for $R \alpha>-\frac{1}{2}$, and recalling the fact that $N_{\alpha}=O\left(e^{(3 \pi+\varepsilon)|\alpha|}\right)$, we have

$$
D_{N_{\alpha}}[\sigma]=O\left(\exp \left(e^{(4 \pi+\varepsilon)|\alpha|}\right)\right)
$$

and the lemma is proved. From (5.5) and the above lemma we know that $E_{\alpha, \beta}$ satisfies a growth condition that is $O\left(\exp e^{(4 \pi+e)|\alpha|}\right)$ for $|R \alpha|<\frac{1}{2}$.

Let us now turn for a moment to the factor $G(1+2 \alpha) / G(1+\alpha-\beta) G(1$ $+\alpha+\beta)$ in $Q(\alpha) \cdot G(1+z)$ has a factorization of the form

$$
e^{p(z)} \prod_{n=1}^{\infty}\left(1+\frac{z}{n}\right)^{n} \exp \left(-\frac{z}{n}+\frac{z^{2}}{2 n}\right)
$$

and here $p(z)$ has degree 2. Using this factorization and [4, Theorem 2.6.18] it is clear that on the strip $-\frac{1}{2}<R \alpha \leqslant \frac{1}{2}$ the above factor has order two.

So at least we can say that from Lemmas 5.2-5.4, $Q(\alpha)$ has an entire logarithm that satisfies

$$
R \log Q(\alpha) \leqslant A\left(e^{(4 \pi+\varepsilon)|\alpha|}\right) .
$$


Using the Carathéodory inequality [4, Theorem 1.3.1] one can conclude that $|\log Q(\alpha)|$ is also $O\left(e^{(4 \pi+\varepsilon)|\alpha|}\right)$. Hence the logarithm is of exponential type. Fortunately there are very few entire functions that are periodic of exponential type. In fact any function $Q(\alpha)$ satisfying these conditions must be of the form [4, Theorem 6.10.1]

$$
\exp \left[a \alpha+b+\sum_{\substack{k=-n \\ k \neq 0}}^{n} c_{k} e^{2 \pi i k \alpha}\right],
$$

where $n$ is less than or equal to the type $\tau$ of the function $\sum_{-n}^{n} c_{k} e^{2 \pi i k \alpha}$. In this case $n$ is clearly $<2$. We now want to eliminate all the coefficients in (5.8). Our first observation is to remark that (5.8) can be written as

$$
\exp \left(a \alpha+b+\sum_{1}^{2} c_{k} \sin 2 \pi k \alpha+\sum_{1}^{2} c_{k}, \cos 2 \pi k^{\prime} \alpha\right)
$$

where all the coefficients now are real. This can easily be seen by observing that $Q(\alpha)$ is real on the real axis and then taking conjugates in (5.8).

In order for (5.9) to be periodic it is necessary for $R a=0$ and, hence, $a=0$. We observe also that (5.9) can be written as

$$
\exp \left(b+\sum_{1}^{2} d_{k} \sin \left(2 \pi k \alpha+\gamma_{k}\right)\right)
$$

where the $d_{k}$ 's are still real and $0 \leqslant \gamma_{k} \leqslant \pi$. Now for $0<R \alpha<\frac{1}{4}$ we have from our estimates for $\left\|H_{n}[\sigma]\right\|_{2},\left\|H_{n}\left[\tilde{\sigma}_{-}^{-1}\right]\right\|_{2}$ and $\left\|T_{n}\left[\sigma_{+}^{-1}\right]\right\|_{\infty}$ in (5.7),

$$
D_{N_{\alpha}}=O\left(\exp e^{\pi|\alpha|} N_{\alpha}^{1 / 4}\right)=O\left(\exp e^{5 \pi|\alpha| / 2}\right),
$$

since $N_{\alpha}=O\left(e^{(3 \pi+e)|\alpha|}\right)$. From the behavior of $\sin (2 \pi k \alpha+\gamma)$ on appropriate lines in the strip $0<R \alpha<\frac{1}{4}$, one can see the above representation is impossible unless $d_{2}=0$, and we can say that (5.9) can be written as

$$
\exp \left(b+c_{1} \sin 2 \pi \alpha+c_{2} \cos 2 \pi \alpha\right) \text {. }
$$

To complete the proof that $Q(\alpha)=1$ and conclude that

$$
E_{\alpha, \beta}=G(1+\alpha+\beta) G(1+\alpha-\beta) / G(1+2 \alpha),
$$

we need to know the value of $Q(\alpha)$ at three points. At the points $\alpha=0,+\beta$, $-\beta$ we can say $Q(\alpha)=1$. The case $\alpha=0$ follows from results of Hartwig and Fisher [6]. The case $\alpha=\beta$ follows from the fact that when $\alpha=\beta$,

$$
T_{n}[\sigma]=T_{n}\left[\left(1-e^{i \theta}\right)^{2 \beta}\right] .
$$

The matrix $T_{n}[\sigma]$ is hence triangular and $E_{\alpha, \beta}=1$. Since at $\alpha=\beta, G(1+\alpha$ $+\beta) G(1+\alpha-\beta) / G(1+2 \alpha)=1$, we have $Q(\alpha)=1$. The same result holds for $\alpha=-\beta$. Therefore we can conclude that $Q(\alpha)=1$ for all $\alpha$ and 


$$
E_{\alpha, \beta}=G(1+\alpha+\beta) G(1+\alpha-\beta) / G(1+2 \alpha) \text {. }
$$

\section{BIBLIOGRAPHY}

1. E. W. Barnes, The theory of the G-function, Quart. J. Pure Appl. Math. 31 (1900), 264-313.

2. H. Bateman, Higher transcendental functions, Vol. 1, Bateman Manuscript Project (A. Erdélyi, Editor), McGraw-Hill, New York, 1953. MR 15, 419.

3. __ Tables of integral transforms, Vol. I, Bateman Manuscript Project (A. Erdélyi, Editor), McGraw-Hill, New York, 1954. MR 15, 868.

4. R. P. Boas, Entire functions, Academic Press, New York, 1954. MR 16, 914.

5. H. Buchholz, The confluent hypergeometric function with special emphasis on its applications; English transl., Springer-Verlag, Berlin and New York, 1969. MR 39 \# 1692.

6. M. E. Fisher and R. E. Hartwig, Toeplitz determinants. Some applications, theorems and conjectures, Adv. Chem. Phys. 15 (1968), 333-353.

7. I. C. Gohberg and M. G. Krein, Introduction to the theory of linear nonselfadjoint operators in Hilbert space; English transl., Transl. Math. Monographs, vol. 18, Amer. Math. Soc., Providence, R. I., 1968. MR 39 \#7447.

8. U. Grenander and G. Szegö, Toeplitz forms and their applications, Univ. of Calif. Press, Berkeley, 1958. MR 20 \# 1349.

9. I. I. Hirschman, Jr., On a formula of Kac and Achiezer, J. Math. Mech. 16 (1966), 167-196. MR 34 \#8089.

10. , On a theorem of Szegö, Kac, and Baxter, J. Analyse Math. 14 (1965), 225-234. MR 31 \#1513.

11. Recent developments in the theory of finite Toeplitz operators, Advances in Probability \& Related Topics, Vol. 1, Dekker, New York, 1971. MR 46 \#4260.

12. A. Leonard, Some remarks on large Toeplitz determinants, Pacific J. Math. 42 (1972), 137-145.

13. G. Szegö, Orthogonal polynomials, Amer. Math. Soc. Colloq. Publ., vol. 23, rev. ed., Providence, R. I., 1959. MR 21 \# 5029.

14. E. T. Whittaker and G. N. Watson, $A$ course of modern analysis, 4th ed., Cambridge Univ. Press, New York, 1962. MR 31 \#2375.

15. H. Widom, Asymptotic behavior of block Toeplitz matrices and determinants. II, Advances in Math. 21 (1976), 1-29.

16. __ Toeplitz determinants with singular generating functions, Amer. J. Math. 95 (1973), 333-383. MR 48 \#9441.

\footnotetext{
Department of Mathematics, California Polytechnic State UniverSITY, SAN LUIS OBISPO, CALIFORNIA 93407
} 